\title{
Internalized Tau Oligomers Cause Neurodegeneration by Inducing Accumulation of Pathogenic Tau in Human Neurons Derived from Induced Pluripotent Stem Cells
}

\author{
Marija Usenovic, Shahriar Niroomand, Robert E. Drolet, Lihang Yao, Renee C. Gaspar, Nathan G. Hatcher, \\ Joel Schachter, John J. Renger, and Sophie Parmentier-Batteur \\ Neuroscience, Merck Research Laboratories, West Point, Pennsylvania 19486
}

Neuronal inclusions of hyperphosphorylated and aggregated tau protein are a pathological hallmark of several neurodegenerative tauopathies, including Alzheimer's disease (AD). The hypothesis of tau transmission in AD has emerged from histopathological studies of the spatial and temporal progression of tau pathology in postmortem patient brains. Increasing evidence in cellular and animal models supports the phenomenon of intercellular spreading of tau. However, the molecular and cellular mechanisms of pathogenic tau transmission remain unknown. The studies described herein investigate tau pathology propagation using human neurons derived from induced pluripotent stem cells. Neurons were seeded with full-length human tau monomers and oligomers and chronic effects on neuronal viability and function were examined over time. Tau oligomer-treated neurons exhibited an increase in aggregated and phosphorylated pathological tau. These effects were associated with neurite retraction, loss of synapses, aberrant calcium homeostasis, and imbalanced neurotransmitter release. In contrast, tau monomer treatment did not produce any measureable changes. This work supports the hypothesis that tau oligomers are toxic species that can drive the spread of tau pathology and neurodegeneration.

Key words: Alzheimer's disease; hiPSC neurons; neurodegeneration; pathology propagation; tau oligomer seeds

\section{Significance Statement}

Several independent studies have implicated tau protein as central to Alzheimer's disease progression and cell-to-cell pathology propagation. In this study, we investigated the ability of different tau species to propagate pathology in human neurons derived from induced pluripotent stem cells, which to date has not been shown. We demonstrated that tau oligomers, but not monomers, induce accumulation of pathological, hyperphosphorylated tau. This effect was accompanied with neurite degeneration, loss of synapses, aberrant calcium homeostasis, imbalanced neurotransmitter release, and ultimately with neuronal death. This study bridges various tau pathological phenotypes into a single and relevant induced pluripotent stem cell neuronal model of human disease that can be applied to the discovery of the mechanisms of tau-induced neurodegeneration.

\section{Introduction}

Abnormal accumulation of tau protein into intracellular hyperphosphorylated aggregates known as neurofibrillary tangles

Received April 19, 2015; revised Aug. 26, 2015; accepted Sept. 11, 2015.

Author contributions: M.U., J.S., J.J.R., and S.P.-B. designed research; M.U., S.N., R.E.D., and L.Y. performed research; M.U., S.N., R.E.D., L.Y., R.C.G., N.G.H., and S.P.-B. analyzed data;M.U., S.N., R.E.D., N.G.H., and S.P.-B. wrote the paper.

We thank Mali L. Cosden, for help with AlphaLISA assay; Cheryl A. Gretzula, for calcium imaging; and other members of Merck Research Laboratories Neuroscience, especially Angela Marie Jablonski, for helpful suggestions and discussions. We also thank Alexis Nazabal and Ryan Wenzel (CovalX, Analytics), for MALDI-TOF MS analysis, and Bob Hepler, for help with atomic force microscopy.

Authors are employees of Merck, and may hold financial interest in the company.

Correspondence should be addressed to either Marija Usenovic or Sophie Parmentier-Batteur, Merck Research Laboratories, 770 Sumneytown Pike, West Point, PA 19486. E-mail: marija.usenovic@merck.com or sophie_parmentier_batteur@merck.com.
(NFTs) is a pathological hallmark of Alzheimer's disease (AD). The principle of tau propagation in $\mathrm{AD}$ has emerged due to temporal and spatial progression of tau pathology observed in AD patients' brains that also correlated with patients' cognitive decline (Arriagada et al., 1992). In addition, recent preclinical studies have shown that exogenously applied misfolded tau protein can be internalized by cells (Frost et al., 2009; Guo and Lee, 2011, 2013; Wu et al., 2013) and can gradually propagate via interconnected brain regions in animal models (Clavaguera et al., 2009, 2013, 2014; Lasagna-Reeves et al., 2012b). Moreover, in vitro studies have shown that tau can be released from different cell lines, including rat primary neurons (Kfoury et al., 2012; Simón 
et al., 2012; Pooler et al., 2013). Therefore, it was hypothesized that transmission of tau pathology is mediated by the release, uptake, and trafficking of pathogenic tau within synaptically connected neurons. Once internalized within cells, pathogenic misfolded tau proteins act as a "seed" that recruits soluble endogenous tau into larger pathological conformations (Jucker and Walker, 2013). A better understanding of the steps of tau transmission and intracellular aggregation could lead to the discovery of novel therapeutic strategies that would inhibit the spread of tau pathology and its consequences, including neurodegeneration and cognitive dysfunction. However, the use of non-neuronal or neuronal in vitro models that overexpress wild-type or mutant tau (Guo and Lee, 2011; Kfoury et al., 2012; Saman et al., 2012; Falcon et al., 2015) may mislead and limit the exploration of these processes.

Using neurons from human induced pluripotent stem cells (hiPSCs), we here developed a cellular model that recapitulates tau pathology and its long-term effect on neuronal degeneration. The model consists of seeding hiPSC-derived neurons with preparations of tau monomers or tau oligomers made from wild-type, full-length recombinant human tau. Tau oligomers are formed before tau assembly into mature insoluble NFTs (Mandelkow and Mandelkow, 2012). We used tau oligomers rather than tau fibrils because these tau species have been identified at the early stages of tau aggregation in the brains of patients with $\mathrm{AD}$ before NFTs can be detected (Maeda et al., 2006; Lasagna-Reeves et al., 2012a). Moreover, accumulation of tau oligomers, before the formation of NFTs, is associated with the development of cognitive and motor deficits during the pathogenic progression of tauopathy in animal models (Berger et al., 2007; Lasagna-Reeves et al., 2011).

The use of human neurons enabled a long-term evaluation of tau-induced toxicity and its relationship to tau pathology, neuronal dysfunction, and neurodegeneration. The human neurons used in this study are a highly purified population of forebrain neurons with active GABAergic and glutamatergic receptors (Dage et al., 2014). These neurons have been previously used to explore the toxicity of $\beta$-amyloid 1-42 (Xu et al., 2013) and tau release (Chai et al., 2012). They can grow and mature in culture for a period of several weeks to months, so they represent a relevant cellular system to study chronic neurodegenerative diseases, such as AD.

In the present study, tau oligomers were shown to be capable of entering human neurons and triggering the formation of aggregated and hyperphosphorylated pathological tau. These effects were associated with long-term defects in neurite outgrowth, synaptic loss, neuronal loss, abnormal neurotransmission, and intracellular calcium mobilization. Whereas neurons treated with tau monomers did not demonstrate alterations in the above measurements.

\section{Materials and Methods}

hiPSC neurons. hiPSC neurons (iCell neurons) were purchased from Cellular Dynamics International (CDI). Fibroblasts from healthy male individuals were reprogramed and differentiated into iCell neurons. These neurons were previously characterized as cortical forebrain human neurons (Haythornthwaite et al., 2012; Xu et al., 2013; Alhebshi et al., 2014; Dage et al., 2014). The cells were seeded on plates precoated with poly-D-lysine and laminin following CDI protocol. The neurons were kept in vitro for $\leq 28 \mathrm{~d}$ with half media change every $7 \mathrm{~d}$. For the examination of the pathological effect of tau seeds, neurons were treated with preparations of tau monomers and oligomers for $24 \mathrm{~h}$, and then extensively washed to remove the nonabsorbed seeds.

Preparation of tau oligomers and fibrils. Recombinant human 4R2N T40 was expressed by bacterial cultures. Cells were harvested following a
10 min centrifugation at $5000 \times \mathrm{g}$. Cell paste was resuspended in $5 \times$ lysis buffer (50 mm Tris-HCl, 1 mm EGTA, 1 mм $\mathrm{MgSO}_{4}, 2$ mм DTT, $750 \mathrm{~mm}$ $\mathrm{NaCl}, 20 \mathrm{~mm} \mathrm{NaF}, 1 \mathrm{~mm}$ PMSF, $20 \mathrm{~mm}$ imidazole and proteinase inhibitor tablet), and lysed using a microfluidizer. Cell lysate was boiled by immersion in boiling water $\left(20 \mathrm{~min}\right.$ at $\left.95^{\circ} \mathrm{C}\right)$, then crash cooled on wet ice. Lysate was then clarified by centrifugation at $40,000 \times g, 1 \mathrm{~h}$ at $4^{\circ} \mathrm{C}$. Tau protein purification was performed by fast protein liquid chromatography on a HiTrap SP HS column. The column was washed with buffer A (20 mм PIPES, pH 6.5, 1 mм EGTA, 1 mm MgSO 4 , 2 mм DTT, $10 \mathrm{~mm} \mathrm{NaCl}, 20 \mathrm{~mm} \mathrm{NaF}, 0.1 \mathrm{~mm}$ PMSF) and eluted with buffer B (buffer A with $1 \mathrm{~mm} \mathrm{NaCl}$ ). The resulting fractions with the highest tau content were pooled and formulated into $100 \mathrm{~mm} \mathrm{Na}$-acetate, $\mathrm{pH}$ 7.0, using a G25 desalting column. Aliquots of recombinant tau were snap-frozen and stored at $-20^{\circ} \mathrm{C}$. To prepare tau oligomers, $5 \mu \mathrm{M}$ recombinant tau, dissolved in MES buffer (4-morpholineethanesulfonic acid hydrate), $\mathrm{pH}$ 6.5, was mixed with $10 \mu \mathrm{M}$ DTT (BioShop) and incubated for $10 \mathrm{~min}$ at $55^{\circ} \mathrm{C}$. Subsequently, $5 \mu \mathrm{M}$ heparin (Fisher, H19) was added to the solution to induce aggregation and incubated with shaking (1000 rpm) for $4 \mathrm{~h}$ at $37^{\circ} \mathrm{C}$. Formation of tau fibrils was also induced in the presence of heparin, but incubation times were increased $(5,10$, and $15 \mathrm{~d})$. Tau monomers, used as a control in this study, were prepared through an identical protocol without the addition of heparin.

Western blot analysis and sarkosyl extractions. For the characterization of tau oligomers by Western blot, the same amounts $(0.1 \mu \mathrm{g})$ of tau monomer and oligomer of $4 \mathrm{~h}$ preparations were separated on $4-12 \%$ NuPAGE Bis-Tris gels (Novex, Life Technologies), without reducing agent. Tau was detected using rabbit anti-tau primary antibody (Dako, A0024;1:4000) and goat anti-rabbit secondary antibody (Li-Cor; $1: 10,000)$. For detection of hyperphosphorylated tau aggregates, iCell neurons were plated on six-well plates $(1,000,000$ cells/well $)$ and treated with 50 and $200 \mathrm{~nm}$ tau oligomers and monomers. On day 6 after treatment, neurons were lysed in 2\% sarkosyl buffer (50 mM Tris, $150 \mathrm{~mm}$ $\mathrm{NaCl}, 1$ mm EDTA, 1 mм EGTA, pH 7.4) with protease and phosphatase inhibitor mixture and subjected to three freeze-thaw cycles. Cell lysates were then incubated for $1 \mathrm{~h}$ with constant shaking $(700 \mathrm{rpm})$ at $4^{\circ} \mathrm{C}$. Lysates were centrifuged at $100,000 \times g$ for $1 \mathrm{~h}$ at $4^{\circ} \mathrm{C}$. Supernatants were collected as sarkosyl-soluble fractions. The pellets were washed in sarkosyl buffer and centrifuged at $100,000 \times g$ for $45 \mathrm{~min}$ at $4^{\circ} \mathrm{C}$. Supernatants were discarded while pellets were resuspended in $2 \%$ SDS buffer, sonicated, and boiled for $10 \mathrm{~min}$. After centrifugation at $16,000 \times g$ for 30 $\mathrm{min}$ at room temperature (RT), supernatants were collected and used as sarkosyl-insoluble fractions. Fractions were analyzed by SDS-PAGE followed by Coomassie blue staining of the gel to visualize total proteins. An immunoblotting was performed using PHF1 (1:1000; from Dr. Peter Davies) and GAPDH (1:500; Millipore) primary antibodies. Odyssey Infrared Imaging System (Li-Cor) and Odyssey software V2.1 was used to scan and analyze the blots.

Determination of tau oligomers by high-mass matrix-assisted laser desorption/ionization time-of-flight mass spectrometry. Tau oligomer and monomer sample sets were shipped to CovalX for analysis by high-mass matrix-assisted laser desorption/ionization time-of-flight mass spectrometry (MALDI-TOF MS). Sample sets included tau monomer preparations $(5 \mu \mathrm{M})$, tau oligomer preparations $(5 \mu \mathrm{M})$, and heparin $(5 \mu \mathrm{M})$. A single sample set was subjected to cross-linking using the CovalX K200 cross-linking kit. Briefly, oligomer complexes were covalently linked at free amine groups via bis-azabenzotriazole ester containing molecules with differing carbon chain lengths (Bich et al., 2010). Tau and heparin solutions were incubated with K200 cross-linking stabilizer for $3 \mathrm{~h}$ at $4^{\circ} \mathrm{C}$, after which both cross-link-treated and nontreated sample sets were subjected to methanol-chloroform liquid-liquid extraction. Tau and heparin precipitates were separated by centrifugation $(10,000 \times g$ for $5 \mathrm{~min})$, and supernatants were removed and dried under vacuum. Dried sample pellets were each reconstituted in $5 \mu \mathrm{l}$ of formic acid and diluted 1:3 into a saturated solution of sinapinic acid MALDI matrix $(10 \mathrm{mg} / \mathrm{ml}$ mixed in a $50 \%$ solution of acetonitrile and $0.1 \%$ trifluoroacetic acid). Samples were spotted at $1 \mu \mathrm{l}$ onto a 384 Anchorchip MALDI target (Bruker) and allowed to crystalize at RT. MALDI-TOF MS analyses were performed following sample crystallization. MALDI is a soft ionization approach that, unlike electrospray ionization, tends to favor the formation of singly 
charged analyte ions, thus simplifying charge state deconvolution in full scan data and subsequent peak identification by mass. Additionally, the mass range of conventional linear time-of-flight instruments can be extended to 1-2 MDa through the incorporation of modified ion detectors, and when used in concert are referred to as high-mass MALDI-TOF MS (Nazabal et al., 2006). MALDI-TOF MS was performed using Reflex IV MALDI-TOF mass spectrometer (Bruker) equipped with an add-on HM2 TUVO high-mass detector (CovalX). Mass spectra were acquired in linear-positive mode at $20 \mathrm{kV}$ with the HM2 detector gain set to 3.24 $\mathrm{kV}$. Mass spectral data represent the summation of 300 laser shots acquired by automated raster positioning within sample spots, and masses are represented as average mass values given linear TOF detection mass resolution limitations. MS data were analyzed using Complex Tracker analysis software (version 2.0, CovalX), and tau and heparin analytes were determined by $m / z$ values in full scan mass spectrum with external calibration.

Atomic force microscopy. A solution of tau monomers and oligomers (5 $\mu \mathrm{M})$ was applied to a freshly cleaved muscovite mica substrate (Ted Pella) and incubated for $1 \mathrm{~min}$. The mica surface was then washed six times with double-distilled water to remove salts and unbound proteins. Atomic force microscopy images were taken on a MultiMode SPM (Digital Instruments) equipped with an e-scanner using etched silicon nanoprobes (Veeco Instruments). All measurements were performed in the tapping mode with scan rates of $\sim 0.5 \mathrm{~Hz}$. Images were processed using NanoScope software (Digital Instruments).

Fluorescence polarization assay. Fluorescence polarization (FP) assay was used to characterize the formation of tau oligomers. This assay required production of small tau fragment K18. This truncated tau consists of the four repeat microtubule-binding region of the wild-type T40 tau (starting from Q244 and ending with E372, numbering according to the amino acid sequence of T40). This region was cloned into the NdeI and EcoRI restriction sites of the bacterial expression vector pET30a. After confirmation of correct reading frame by DNA sequencing, the tau protein was purified as previously described (Li and Lee, 2006). Five nanomole K18-tau labeled with Texas red (Alexa Fluor 594, Life Technologies) was spiked into the tau aggregation reaction (described above) to measure a change in FP at different times, which represents the binding kinetics of truncated K18 to full-length T40 tau. The change in FP [ $\Delta$ millipolarization value $(\Delta \mathrm{mp})]$ was measured between reaction mixture with heparin (oligomerization reaction) and without heparin (negative control monomer) at each time point (0, 2, 4, 6, 8, $24 \mathrm{~h}$ ) using an EnVision multiplate reader (PerkinElmer) equipped with the Texas Red FP filter and dichroic mirror sets.

Thioflavin T-binding assay. Thioflavin T (ThT; Sigma-Aldrich) dye fluorescence was used to quantify the formation of tau fibrils. The change in ThT fluorescence was measured at different time points $(0,1,5,10$, and $15 \mathrm{~d}$ ) after the preparation of tau oligomer seeds kept under agitation at $37^{\circ} \mathrm{C}$ as described above. Briefly, in a black 96-well plate, $10 \mu \mathrm{l}$ of tau seed preparation was added to $90 \mu \mathrm{l}$ of ThT diluted in $100 \mathrm{~mm}$ glycine such that the final concentration of ThT was $5 \mu \mathrm{M}$ when mixed with sample. The reaction was thoroughly resuspended to avoid sedimentation and clumping of the filaments. The intensity of fluorescence was measured using a SpectraMax fluorometer (excitation, $450 \mathrm{~nm}$; emission, $550 \mathrm{~nm}$; cutoff, $475 \mathrm{~nm}$ ).

Internalization assay of Cy3-tau conjugates. Tau monomers were conjugated to Alexa Fluor 555 (Cy3) with final degree of labeling 3.2 (3.2 moles of dye/mole of protein) (Life Technologies, outsourcing facilities). From Cy3-tau monomers, we produced oligomers and fibrils ( $10 \mathrm{~d}$ preparation) using the protocol described above. For internalization assay, hiPSC neurons on day in vitro (DIV) 7 were treated for $1 \mathrm{~h}$ with $50 \mathrm{~nm}$ labeled tau monomers, oligomers, or fibrils at $37^{\circ} \mathrm{C}, 5 \% \mathrm{CO}_{2}$, in a medium with Hoechst 33342 (Life Technologies; 1:1000) to label nuclei. After $1 \mathrm{~h}$ of incubation, cells were rinsed two times followed by addition of red background suppressor (Life Technologies). Immediately after, live-cell imaging was performed using the Operetta high-content imaging system (PerkinElmer) with a $20 \times$ objective. To test the effect of heparinase III (Sigma-Aldrich, H8891, SLBN5069V) on tau uptake, hiPSC neurons were treated with the $1.5 \mathrm{mIU} / \mathrm{ml}$ enzyme for $3 \mathrm{~h}$ before the addition of Cy3-tau oligomers and together with the oligomers for $1 \mathrm{~h}$.

Microfluidics and tau trafficking. Microfluidic standard neuronal devices (with $450 \mu \mathrm{m}$ microgroove barriers; Xona Microfluidics) were handled as suggested by the manufacturer's protocol. Each device was mounted on cover glass $(24 \times 24 \mathrm{~mm}$, Gold Seal, \#1) coated with polyD-lysine $(0.5 \mathrm{mg} / \mathrm{ml}$, Sigma-Aldrich). Before cell plating, each assembled device was additionally coated with laminin $(3.3 \mu \mathrm{g} / \mathrm{ml}$; Sigma-Aldrich). Twenty microliters of cell suspension (2-3 million cells $/ \mathrm{ml}$ ) were added to one compartment of each device (somal side). To detect exogenous tau, monomers were conjugated with aminoreactive fluorescent dye O-succinimidylester, Alexa Fluor 647 (Cy5), with the final degree of labeling 3.7 (3.7 moles of dye/mole of protein) (Life Technologies, outsourcing facilities). From Cy5-monomers we produced Cy5-oligomers. On DIV 10, once the axons fully crossed the microgrooves $(450 \mu \mathrm{m}$ distance) into the axonal compartment of a device, $5 \mu \mathrm{l}$ of $5 \mu \mathrm{M}$ Cy5-tau oligomers or monomers was added into the somal side for $2 \mathrm{~h}$. A $60 \mu \mathrm{l}$ difference in media volume was maintained to prevent spontaneous diffusion of Cy5-tau species from the somal to the axonal compartment. Following $2 \mathrm{~h}$ of incubation, cells were washed and subsequently imaged after 18 h using fluorescence microscope at $40 \times$ objective (EVOS FL, Life Technologies).

Immunocytochemistry. hiPSC neurons were plated in 96-well plates (Corning) at the density of 25,000 cells/well, which was the optimal density for the both long-term survival of iCell neurons and for the accuracy of high-content image analysis. At indicated time points after tau treatment, cells were fixed in $4 \%$ paraformaldehyde for $15 \mathrm{~min}$ at RT followed by three washes with Dulbecco's PBS (DPBS). Fixed cells were kept in DPBS and stored at $4^{\circ} \mathrm{C}$ until immunocytochemistry staining. Cells were incubated in blocking and permeabilization buffer $(0.2 \%$ Triton X-100, 2\% goat serum, and 0.1\% BSA in DPBS; all Sigma-Aldrich) for $1 \mathrm{~h}$ shaking at RT. After blocking, cells were incubated with primary antibodies diluted in antibody solution (2\% goat serum, $0.1 \%$ BSA in DPBS) overnight at $4^{\circ} \mathrm{C}$. The day after, plates were washed three times $(5$ min each) with DPBS (200 $\mu \mathrm{l} /$ well). The following antibodies were used for immunostaining: MC-1 (from Dr. Peter Davies; $2 \mu \mathrm{g} / \mathrm{ml}$ ), CP17 (from Dr. Peter Davies, phospho-T231; $2 \mu \mathrm{g} / \mathrm{ml}$ ), PHF1 (from Dr. Peter Davies, phospho-S396/404; $2 \mu \mathrm{g} / \mathrm{ml}$ ), total tau (Dako, A0024; 1:2000), microtubule-associated protein 2 (MAP2; Millipore, AB5622; 1:100), $\beta$-III-tubulin (TUJ-1; neuron-specific class III $\beta$-tubulin, Covance, PRB435P; 1:1000), synapsin (Synaptic Systems, 106 001; 1:500), synaptophysin (Synaptic Systems, 101 011; 1:100), LC3 (Cell Signaling Technology, 2775; 1:50), HT7 (ThermoScientific, MN1000; 1:1000), and anti-Flag (Sigma-Aldrich, F7425; 1:500). Staining for heparan sulfates was performed without cell permeabilization using anti-heparan sulfate antibody (USBiological, H1890; 1:50). Secondary antibodies (Invitrogen, A11029, A21245; A10680) diluted in antibody solution (1:600) were incubated for $1 \mathrm{~h}$ at RT, and subsequently washed three times in DPBS. Hoechst 33342 solution was used to stain nuclei (Anaspec, 83218; 1:5000 in DPBS; $100 \mu \mathrm{l} /$ well). High-content imaging was performed using an Operetta system (PerkinElmer) with a $20 \times$ objective. To examine the colocalization of HT7, anti-Flag staining, and localization of MC1positive tau aggregates, hiPSC neurons were plated on coverslips and imaged with Nikon TE2000-U eclipse microscope with a PerkinElmer UltraViewERS Nipkow Spinning Disc Confocal imaging system, using Volocity acquisition software.

Thioflavin S staining. Cells previously stained with total tau (Dako, A0024; 1:2000) were permeabilized with $0.1 \%$ Triton X-100 for 15 min and washed two times with PBS. Thioflavin S (ThS) $0.01 \%$ (SigmaAldrich) was added for $5 \mathrm{~min}$ at RT, followed by washing steps in $70 \%$ ethanol $(1 \times 10 \mathrm{~min}$, then $2 \times 5 \mathrm{~min})$ and final addition of PBS. Highcontent imaging was performed using an Operetta system (PerkinElmer) with a $20 \times$ objective.

High-content image analysis. The Operetta system was used for the high-content imaging. To cover a good portion of the surface of the well, $\geq 9$ fields per well were imaged, analyzed, and averaged. A total of 3-6 wells was used per treatment condition $(n=3-6)$ and each experiment was repeated $\geq 3$ times. Image analyses and calculations were performed using Harmony 3.1.1 software (PerkinElmer). Hoechst staining was used 
to label cell nuclei. Single-cell identification was performed using the cell mask algorithm based on total tau staining that labeled both the cell cytoplasm and neurites. Within the cell mask, we quantified the average intensity of MC1, PHF1, CP17, or ThS staining. To investigate the neuronal population affected by tau pathology, we counted the cells positive for the above markers. Cells were determined positive if they presented a fluorescence intensity of markers staining higher than a defined threshold. The threshold was established based on the distribution of the fluorescence intensity measured in vehicle-treated cells. Defined thresholds calibrated and standardized the analyses based on the background fluorescence of $\mathrm{MCl}$ and ThS staining, and also on the endogenous levels of phospho-tau labeling with PHF1 and CP17. Results were expressed as percentage of positive cells per well. Neuronal morphology was analyzed using neurite outgrowth module of Harmony 3.1.1 software (PerkinElmer), which detected all neurites. Neurites were detected based on total tau, TUJ-1, and MAP2 immunostaining. These markers enabled detection of neuronal processes, including both axons and dendrites. Nuclei detected based on Hoechst staining were enlarged to cover the size of neuronal cell body and to reach the neurites attached to each individual cell body. A neurite mask was refined to detect all neurites starting from enlarged nuclei (see Fig. 5A). Total neurite length and number of segments, defined as part of the neurites between neurite intersecting points, were analyzed per cell and averaged per well. To examine the synaptic integrity, we counted the number of puncta per cell identified by either synapsin I or synaptophysin staining located on tau-labeled neurites (used as a mask). Number of autophagosomes was analyzed by counting the number of LC3-positive puncta per cell.

Time-lapse imaging and neurite outgrowth analysis. For long-term, continuous, neurite-outgrowth analysis, hiPSC neurons plated in 96well plates were incubated in an IncuCyte Zoom live-cell imaging instrument (Essen Bioscience). Images of four fields per well were acquired every $6 \mathrm{~h}$ for $19 \mathrm{~d}$ (starting at DIV 3 till $14 \mathrm{~d}$ after seeding). For analyses, NeuroTrack software (Essen Bioscience) was used to automatically define neurite processes and cell bodies based on phase contrast (see Fig. $6 A$ ). Total neurite length (in millimeters) and number of branch points were quantified and normalized to the image field area (in square millimeters). Total neurite length presents the summed length of neurites that extend from the cell bodies and number of branch points presents the number of intersections of the neurites in image field.

AlphaLISA assay. An AlphaLISA assay was developed to detect the recruitment of endogenous neuronal tau by the exogenous tau seeds in forming intracellular tau aggregates. For this experiment, tau seeds were prepared using the full-length T40 human tau protein $(4 \mathrm{R} 2 \mathrm{~N})$ that was mutated for the epitope of anti-tau HT7 monoclonal antibody $(\mathrm{P} 159 \mathrm{~S}+\mathrm{G} 161 \mathrm{~A})$ and tagged with Flag-6xHis. The introduction of the Flag tag and the mutation in the epitope for the HT7 monoclonal antibody enabled us to differentiate exogenous tau seeds detected with antiFlag antibody from endogenous tau detected with HT7 antibody. hiPSC neurons plated in 12-well plates (300,000 cells/well) were treated at DIV 7 with two concentrations of tau- $\Delta$ HT7-Flag oligomers and tau- $\Delta$ HT7Flag monomers (50 and $250 \mathrm{~nm}$ ). On day 4 after seeding, cells were lysed in PhosphoSafe Extraction Reagent (Novagen) with protease inhibitor mixture (Roche) and centrifuged at $15,000 \times g$ for $15 \mathrm{~min}$ at $4^{\circ} \mathrm{C}$. Ten microliters of cell lysates were incubated for $2 \mathrm{~h}$ at RT with $20 \mu \mathrm{l}$ of HT7-acceptor beads $[0.05 \mu \mathrm{g} / \mu \mathrm{l}$ final in $50 \mu \mathrm{l}$; HT7 (ThermoScientific, MN1000) conjugated in house] and $20 \mu$ l of Flag-biotinylated antibody (0.15 $\mu \mathrm{l} /$ well; Cell Signaling Technology) in a 96-well OptiPlate (PerkinElmer). AlphaLISA immunoassay buffer (PerkinElmer) was used as a dilution buffer. Last, $50 \mu \mathrm{l}$ of diluted AlphaScreen streptavidincoated donor beads $(40 \mu \mathrm{g} / \mathrm{ml}$ final in $100 \mu \mathrm{l}$; PerkinElmer, 6760002) were added and incubated for $1 \mathrm{~h}$ at RT while gently shaking. Luminescence signal was measured using an EnVision multiplate reader (PerkinElmer).

Meso Scale Discovery sandwich ELISA. To confirm that recombinant tau protein with a mutation in the epitope for HT7 antibody cannot be recognized with HT7 antibody, we performed Meso Scale Discovery (MSD) sandwiched ELISA (Meso Scale Diagnostics). High-bind multiarray 96-well MSD plates (Meso Scale Diagnostics, L15XB-3) were coated overnight at $4^{\circ} \mathrm{C}$ with $25 \mu \mathrm{l} /$ well solution of anti-human tau an- tibody (Dako, A0024) diluted 1:1000 in PBS. The day after, plates were washed three times with MSD wash buffer (Meso Scale Diagnostics, R61TX-1) and incubated with $150 \mu \mathrm{l} /$ well of casein blocking buffer (ThermoScientific, 37528) for $2 \mathrm{~h}$ at RT. After wash with MSD buffer, plates were incubated with different concentrations of recombinant tau proteins diluted in blocking buffer ( $25 \mu \mathrm{l} /$ well $)$ for $1 \mathrm{~h}$ at RT, followed by three washes with MSD buffer. Plates were incubated for $1 \mathrm{~h}$ at RT while being shaken with HT7 antibody (ThermoScientific, MN1000) diluted 1:1000 in blocking buffer, followed by three washes with MSD buffer. Incubation with secondary detection antibody (Sulfotag, MSD R32AC-1; anti-mouse antibody) diluted 1:1000 in blocking buffer was performed at RT while shaking for $1 \mathrm{~h}$. Plates were washed four times with MSD wash buffer and MSD read buffer was added last (Meso Scale Diagnostics, R29TC-3; $150 \mu \mathrm{l} /$ well). Quantification was performed using the MSD plate reader.

Cell viability and toxicity assays. To examine cell viability, cells were treated with calcein AM (Life Technologies), a live cell-permeable dye that produces green fluorescence when cleaved with intracellular esterase. Fluorescence intensity was analyzed in the cells detected with Hoechst 33342 using a high-content image analysis program (PEHarmony 3.1.1 software, PerkinElmer). Results were expressed as dyeintensity percentage of vehicle-treated cells. Small Hoechst-positive spots, which do not likely represent cell nuclei, were excluded from the analysis. Cell toxicity was quantified based on the measurement of activity of lactated dehydrogenase (LDH) in cellular supernatant using the LDH Cytotoxicity Detection Kit (Roche).

Quantification of neurotransmitter release. The measure of the neurotransmitter release was performed in hiPSC neurons (24-well plates; 150,000 cells per well) $14 \mathrm{~d}$ after the addition of tau oligomer seeds. Cell media was replaced with warm cell buffer $(130 \mathrm{~mm} \mathrm{NaCl}, 5 \mathrm{~mm} \mathrm{KCl}, 20$ mM HEPES, 5 mм NaHCO $3,1.2 \mathrm{~mm} \mathrm{Na} 2 \mathrm{HPO}_{4}, 1 \mathrm{~mm} \mathrm{MgCl} 2,100 \mathrm{~nm}$ glycine, $10 \mathrm{~mm}$ glucose, $2.5 \mathrm{~mm} \mathrm{CaCl}_{2}$ ) for $45 \mathrm{~min}$. After this first incubation, the buffer was replaced with a fresh cell buffer (complemented with $100 \mathrm{~nm}$ NO-711 to block GABA reuptake) and cells were incubated for an additional $3 \mathrm{~min}$. The buffer was collected for analysis of extracellular GABA and glutamate concentrations. GABA and glutamate concentrations were quantified using a Waters Acquity UPLC system coupled with tandem MS (MS/MS). The sample (30 $\mu \mathrm{l})$ was first derivatized using the Waters Amino Acid ACCQ Tag Ultra Kit (Waters) based on manufacturer's suggested procedure with a slight modification. The derivatized sample was dried down using Speed Vac followed by reconstitution to $15 \mu \mathrm{l}$ using mobile phase A. Ten microliters of the sample were used for separation using a Waters ACCQ Tag Ultra C18 $(2.1 \times 100$ $\mathrm{mm} ; 1.7 \mu \mathrm{m}$ ) column (Waters) with a gradient mobile phase containing Waters ACCQ Tag Ultra solution A (10\% used in actual sample analysis) and $\mathrm{B}$. The mobile phase was held on the A/B ratio of 99.9:0.1 for 0.54 min. The linear gradient was changed over the next 5.2 min to an $\mathrm{A} / \mathrm{B}$ ratio of 90.9:9. The gradient was further changed to $A / B$ ratio of 78.8:21.2 over next $2 \mathrm{~min}$, followed by another change to A/B ratio of 40.4:59.6 for $0.26 \mathrm{~min}$. The gradient was then changed and held at $\mathrm{A} / \mathrm{B}$ ratio of 10:90 for next $0.6 \mathrm{~min}$ before being returned to initial condition at $\mathrm{A} / \mathrm{B}$ ratio of 99.9:0.1 for $1.4 \mathrm{~min}$. The total run time was $10 \mathrm{~min}$, and flow rate was 0.5 $\mathrm{ml} / \mathrm{min}$. MS/MS detection was performed on an API4000 mass spectrometer (AB Bioscience Instruments) in the positive ion mode (electrospray ionization) by multiple-reaction monitoring (GABA m/z, 274/ 171). Deuterated GABA (d6 GABA; $m / z, 280 / 171$ ) was used as the internal standard. Concentration of GABA was determined by comparing the ratio of peak areas for unknowns versus internal standard (d6 GABA) to standard curves generated from known amounts of GABA (using ratio of standard peak areas vs internal standard). Linear regression curves were plotted for GABA. Absolute concentration of GABA is calculated based on linear regression curve.

Calcium imaging. The measure of intracellular calcium levels was performed in hiPSC neurons (96-well plates, 25,000 cells per well) $14 \mathrm{~d}$ after the addition of tau oligomer seeds. Neurons were incubated for $2 \mathrm{~h}$ with a calcium-sensitive dye (EarlyTox Cardiotoxicity Kit, Molecular Devices) and Hoechst 33342 nuclear stain (Life Technologies; $1: 1000$ ) at $37^{\circ} \mathrm{C}, 5 \%$ $\mathrm{CO}_{2}$ in a cell buffer [HBSS (without $\mathrm{Ca}^{2+}$ and $\mathrm{Mg}^{2+}$ ), 20 mM HEPES, 2 $\mathrm{mm} \mathrm{CaCl}_{2}, 5 \mathrm{~mm}$ D-glucose, $\left.0.1 \% \mathrm{BSA}\right]$. Neurons were treated with $30 \mu \mathrm{M}$ 
A

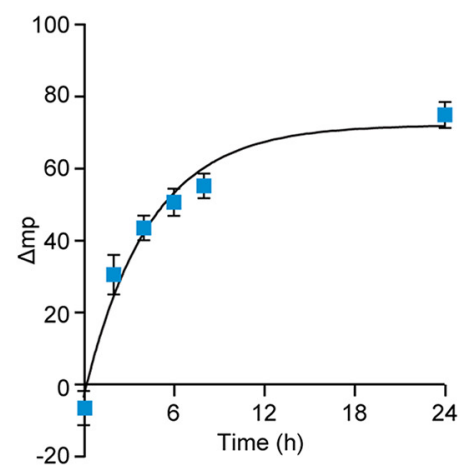

D

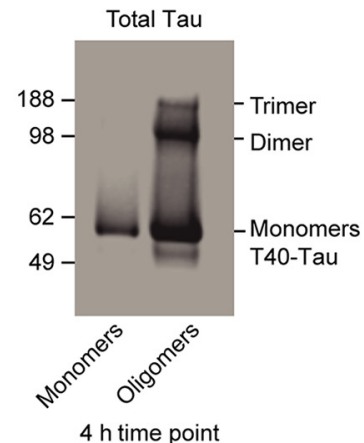

$4 \mathrm{~h}$ time point
B

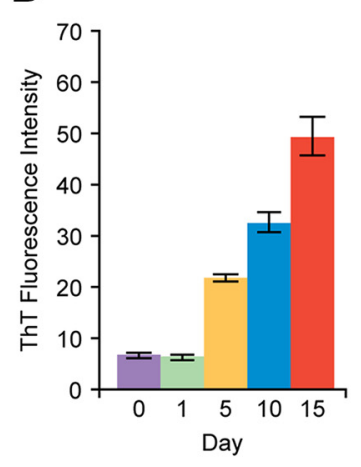

E

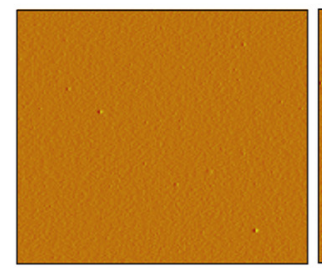

$4 \mathrm{~h}$, Tau monomers

(without heparin)
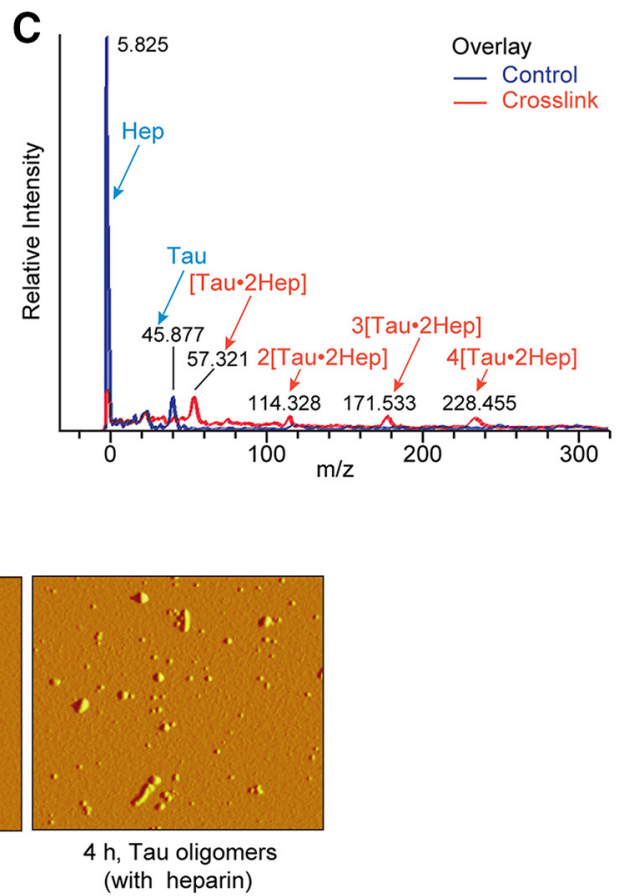

Figure 1. Characterization of tau oligomers. $\boldsymbol{A}$, Graph represents a time course of formation of tau oligomers based on change of FP. Results are expressed as a change of FP ( $\Delta$ mp) between oligomerization reaction (with heparin) and negative control reaction (without heparin) at indicated time points. $\boldsymbol{B}$, Fluorescence intensity of ThT bound to T40-tau during the aggregation process of $15 \mathrm{~d}$ in presence of heparin that led to a tau fibril formation. All values are expressed as mean \pm SEM. C, High-mass MALDI-TOF MS comparing cross-link-stabilized (red) and untreated (blue) tau oligomers demonstrates that our preparations consist of a heterogeneous, multimeric population of tau monomers, dimers, trimers, and tetramers that include noncovalent binding with heparin. $\boldsymbol{D}$, Immunoblot probed with total tau antibody shows the presence of tau monomers, dimers, and trimers in our oligomerization reaction of $4 \mathrm{~h}$. $\boldsymbol{E}$, Images of atomic force microscopy of $4 \mathrm{~h}$ oligomer preparation show the formation of majority spherical oligomeric structures or elongated tau threads. Tau monomers (from the preparation that contains no heparin and therefore does not cause oligomerization) do not bind to mica surface.

NMDA for 10 min (Sigma-Aldrich). High-content imaging of live cells was used to detect the fluorescence produced by the $\mathrm{Ca}^{2+}$ dye (Operetta, PerkinElmer). Image analysis consisted of quantifying the intensity of $\mathrm{Ca}^{2+}$ dye per cell identified by Hoechst 33342 nuclear staining (PEHarmony 3.1.1 software, PerkinElmer).

Statistical analysis. All data were expressed as mean \pm SE. Statistical analyses were performed using Student's $t$ test for comparison between two groups or one-way ANOVA followed by post hoc Dunnett's test for multiple comparisons. GraphPad Prism 6 software was used to perform statistical analyses.

\section{Results}

\section{Production and characterization of tau oligomers}

Recent data suggest that small tau oligomeric species might be involved in the spread of tau pathology and neurotoxicity (Kayed et al., 2003; Lasagna-Reeves et al., 2011, 2012b; Patterson et al., 2011). Therefore, to study tau transmission and propagation we tested the seeding abilities of recombinant full-length tau oligomers in hiPSC neurons, using recombinant full-length tau monomers as controls. Most protocols for tau oligomer preparation use cofactors, such as polyanions or fatty acids, to induce aggregation and the formation of $\beta$-sheet structures around tau microtubule-binding repeat domains (MTBDs). These preparations led to the formation of synthetic fibrils similar to those detected in the brain of AD patients (Gerson and Kayed, 2013). Such fibrillar aggregates can be detected using the fluorophore thioflavin that upon binding to $\beta$-sheet-rich structures displays enhanced fluorescence intensity (LeVine, 1999; Santa-María et al., 2006). Thioflavin has been reliably used to measure protein aggregation across a number of proteinopathies. However, a lim- itation of this detection method is that it is unable to measure the initial kinetic stages of aggregation, such as the oligomerization of amyloidogenic proteins.

To overcome this problem, we developed a tau oligomerization assay using FP-based detection, a method routinely used in high-throughput screening applications for the measurement of ligand binding. The FP method is based on the detection of a small fluorescently labeled peptide binding to a protein of interest. This binding results in detectable FP, which is proportional to the fraction of bound ligand (Jameson and Seifried, 1999; Jameson and Croney, 2003; Moerke, 2009).

In our experiments, the truncated form of tau consisting of the MTBD K18 was the small ligand labeled with Alexa 594 fluorophore, while the binding protein of interest was full-length $4 \mathrm{R} 2 \mathrm{~N}$ T40. When unbound monomeric K18-tau-Alexa 594 is excited by polarized light at the appropriate wavelength, the ligand reorients to a significant degree due to molecular tumbling, causing the emitted light to be largely depolarized. However, when K18-Alexa 594 is bound to the higher molecular weight T40 tau, during the process of oligomerization, the resulting larger complex tumbles much more slowly, and emitted light retains polarization. To induce tau oligomerization, heparin was added to the preparation mixture. We measured a difference in FP $(\Delta \mathrm{mp})$ between the oligomerization reaction (with heparin) and the negative control (without heparin) at each indicated time point of the aggregation process (Fig. $1 A$ ). This approach enabled us to measure the binding kinetics of K18 to tau T40 and to follow oligomerization. The signal revealed a rapid formation of tau 
oligomers during the first $6 \mathrm{~h}$ of reacting with heparin, and then the process of oligomerization stabilized while the formation of fibrils began. In contrast to the rapid detection of tau oligomers by FP, the formation of fibrillar tau cannot be detected using ThT fluorescence until after $1 \mathrm{~d}$. Increased ThT binding was shown $5 \mathrm{~d}$ after the initiation of tau aggregation by heparin and increased further at 10 and $15 \mathrm{~d}$ (Fig. $1 B$ ).

Since FP measures have inherent limitations in terms of distinguishing the specific tau oligomer species present in solution, we used a combination of other approaches to characterize the tau oligomeric species present in our heparin-induced tau oligomers. These approaches included MS, atomic force microscopy, and Western blot analyses.

MALDI-TOF MS offers a number of advantages in the direct analysis of macromolecules, such as protein complexes (Nazabal et al., 2006). Noncovalent interactions do not remain intact during MALDI ionization process, and thus analysis of protein complexes by high-mass MALDI-TOF MS requires chemical cross-linking stabilization at the stage of sample preparation (Bich et al., 2010). Figure $1 C$ overlays high-mass MALDI TOF mass spectra comparing cross-link-treated and untreated tau oligomers formed following $4 \mathrm{~h}$ incubation with heparin. Untreated oligomers readily dissociate during ionization, thus analytes observed represent single molecules of heparin, observed at 5.83 $\mathrm{kDa}$, and tau monomer, observed at $45.88 \mathrm{kDa}$; notably, no gasphase ion clustering artifacts are observed. Mass spectra obtained from cross-link-stabilized tau oligomers show the presence of tau monomers, dimers, trimers, and tetramers. Additionally, the mass values observed for each tau monomer and oligomer complex are elevated by increments consistent with the mass equivalents of two heparin molecules, indicative of a heparintau-specific binding stoichiometry of 2:1. Additional analyses comparing cross-link-stabilized and untreated tau monomer preparations did not detect the presence of tau oligomer analytes (data not shown).

The Western blot analysis of tau oligomers ( $4 \mathrm{~h}$ preparation) demonstrated the heterogeneous population of tau species, as observed by the presence of three bands of molecular weight for tau monomers, dimers, and trimers (Fig. 1D). Images of atomic force microscopy further confirmed the structures of the tau oligomer preparation (Fig. 1E). While the monomeric tau protein would not bind to the mica surface, the oligomeric species taking the shapes of either compact spheres or elongated threads were found at the $4 \mathrm{~h}$ time point. Together, these data demonstrate that tau seeds prepared by $4 \mathrm{~h}$ of aggregation in the presence of heparin contained a mixture of tau oligomers.

\section{Tau monomers and oligomers were internalized and transported along the axons}

To investigate whether tau monomers, oligomers, or fibrils are internalized by hiPSC neurons, tau seeds were prepared using tau recombinant protein conjugated to Cy3-fluorophore. Extracellular Cy3-fluoresence signal was fully quenched by the noncellpermeable red background suppressor (Fig. 2B), allowing detection of only internalized Cy3-tau. Live-cell imaging of neurons at $1 \mathrm{~h}$ after seeding with Cy3-tau seeds showed intense intracellular fluorescence in cells treated with monomers and oligomers, but not in cells treated with tau fibrils, reflecting the uptake of both monomers and oligomers, but not tau fibrils (Fig. $2 A$ ). Recently, it was shown that reduction of heparan sulfate proteoglycans (HSPGs) with enzymatic treatment of heparinase III decreased the internalization of aggregated tau species, suggesting that cellular uptake of tau occurs via HSPG-mediated macropinocytosis (Holmes et al., 2013). Interestingly, the uptake of Cy3-tau oligomers was significantly reduced by the cell treatment with heparinase III for $3 \mathrm{~h}$ before and during the $1 \mathrm{~h}$ exposure to tau seeds (Fig. 2C). Treatment with heparinase significantly reduced the fluorescence of intracellular Cy3-tau oligomers $(p<0.0001$; Fig. $2 C$ ), without causing neuronal loss (Fig. $2 D)$. This further suggests the involvement of HSPGs in tau internalization and supports use of the Cy3-tau conjugate, with a background suppressor, as a tool to study tau uptake. Analysis of the area positive for heparan sulfate immunostaining showed that heparinase III successfully reduced the heparan sulfates from neuronal surface [vehicle: $591.35 \pm 10.43 \mu \mathrm{m}^{2} /$ cell; heparinase III $(1.5 \mathrm{mIU} / \mathrm{ml}): 397.14 \pm 9.21 \mu \mathrm{m}^{2} / \mathrm{cell}, p<$ 0.005 , Student's $t$ test].

To examine the intracellular distribution of tau seeds within the neuronal soma and axonal projections, we seeded tau monomers and oligomers labeled with Cy5-fluorophore on hiPSC neurons plated inside a microfluidic device. The microfluidic device enables neuronal cell bodies to be in a different chemical environment than their axons via hydrostatic pressure, thus preventing the spontaneous diffusion of molecules from somal to axonal compartment (Taylor et al., 2005; Wu et al., 2013). Figure $2 E$ demonstrates the fluidically isolated compartments. Note that there was no leakage of Cy5-tau monomer fluorescent signal from compartment 1 into the microgrooves of the device, confirming the efficacy of the hydrostatic pressure to isolate the two regions (Fig. 2E).

Tau seeds were applied to the microfluidic cultures in the somal compartment after the neurons' axons transversed the entire length of the device's microgrooves (DIV 10). Using live-cell imaging $18 \mathrm{~h}$ after treatment with tau seeds, fluorescent Cy5 granular signal in the axons was detected, suggesting that both tau monomer and oligomeric seeds were internalized and actively transported along the axons (Fig. $2 F$ ). The fluorescence intensity from the axonal compartment of neurons receiving the monomeric seeds was weaker compared with the signal coming from the axons after treatment with oligomeric seeds. This difference in the signal may be due to the lower fluorescence intensity of Cy5-conjugated monomeric seeds compared with oligomer seeds, since the oligomer seeds may incorporate the label with higher density and may therefore be easier to detect.

\section{Internalized tau oligomer seeds but not monomer seeds induced pathological tau conformation and aggregation, and enhanced tau phosphorylation in hiPSC neurons}

Having shown that exogenous tau monomers and oligomers were internalized by neurons (Fig. $2 A, F$ ), we further investigated the effect of these tau seeds on tau conformation and aggregation. Neurons were seeded with $50 \mathrm{~nm}$ tau monomer seeds or tau oligomer seeds for $24 \mathrm{~h}$, and then washed with media. After $7 \mathrm{~d}$, cells were stained with MC1 antibody, a marker of pathological tau conformation (Jicha et al., 1997; Weaver et al., 2000), and ThS, a marker for tau aggregation (LeVine, 1999; Santa-María et al., 2006). High-content imaging analysis was used to measure the percentage of cells positive for these markers. The percentage of MC1 and ThS-positive cells significantly increased (11.70 \pm 1.42 -fold increase in MC1 cells, $p<$ $0.0001 ; 2.24 \pm 0.25$-fold increase in ThS cells, $p<0.005)$ in the cells treated with tau oligomer seeds compared with vehicle-treated cells. There was no change between the tau monomer-treated and vehicletreated cells (Fig. $3 A, B$ ).

We also measured tau phosphorylation levels, which is another indicator of tau pathology (Ballatore et al., 2007; Mandelkow and Mandelkow, 2012). Intracellular intensity of the 

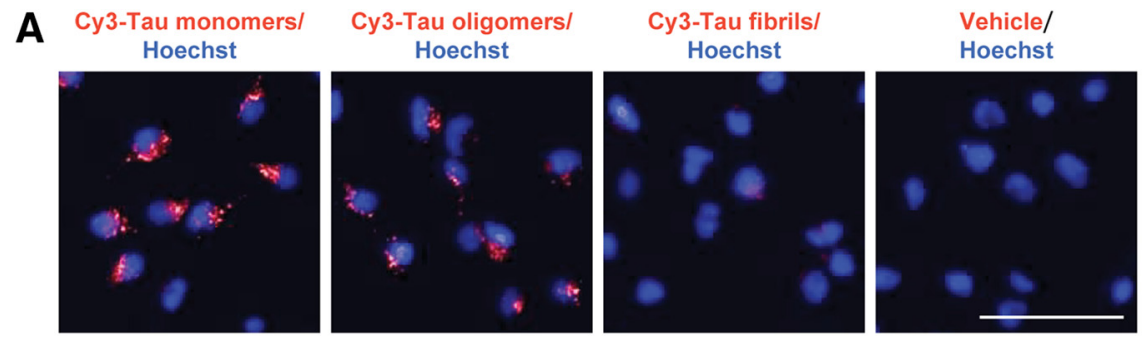

B
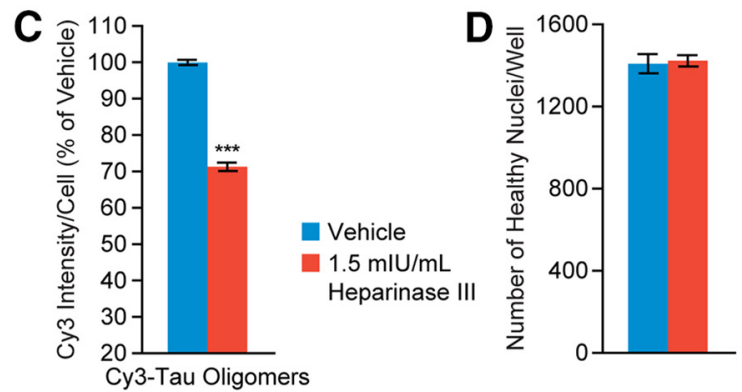

E

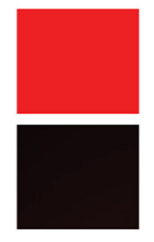

Cy3-Tau

(50nM)

Cy3-Tau

$(50 n M)+$ red

background

suppressor

$\mathbf{F}$

Cy5-Tau Monomers
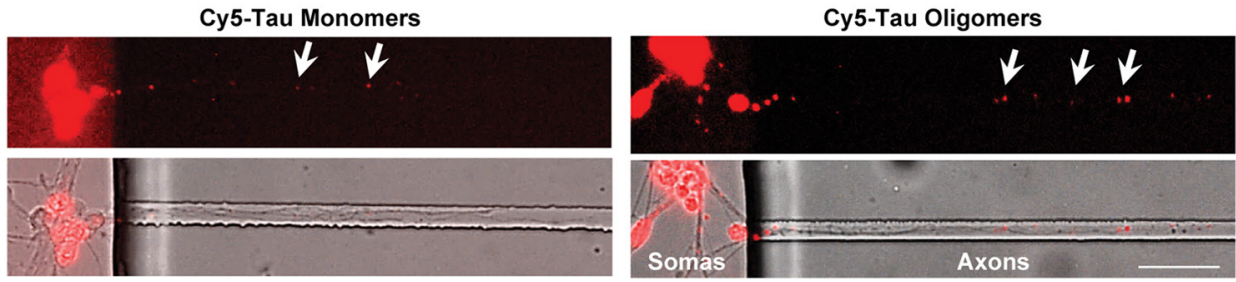

Figure 2. Internalization of tau seeds by hiPSC neurons. $\boldsymbol{A}$, Representative live-cell images of Cy3-tau conjugate (red) show the internalization of tau monomers and oligomers, but not fibrils (10 $\mathrm{d}$ preparation), $1 \mathrm{~h}$ after addition. Hoechst was used to stain nuclei (blue). Scale bar, $50 \mu \mathrm{m}$. B, Images demonstrate the ability of background suppressor to efficiently suppress the fluorescence of Cy3-tau oligomers added to a well with a media, at the same concentration used in the uptake assay. C, Graph shows the decrease in the cellular intensity of Cy3-tau oligomers after heparinase III treatment. hiPSC neurons pretreated for $3 \mathrm{~h}$ with heparinase III were seeded with Cy3-tau oligomers for $1 \mathrm{~h}$ (Student's $t$ test, ${ }^{* * *} p<0.0001$ ). D, Number of healthy nuclei per well with and without heparinase III treatment ( $n=6$ wells per condition, 9 fields, Student's $t$ test, $p=0.8$ ). All values are expressed as mean \pm SEM. $\boldsymbol{E}$, Images of the microfluidic device show the localization of $C y 5$-tau fluorescence signal in compartment 1 of the device where the Cy5-tau was added, but not in the microgrooves, demonstrating that the fluidic isolation creates independent microenvironment in microfluidic compartments. $\boldsymbol{F}$, Images of hiPSC neurons growing in microfluidic device with somas and dendrites localized to a somal compartment and axons crossing the microgrooves. Cy5-tau monomers or oligomers were added to somal compartment for $2 \mathrm{~h}$ and images were taken $18 \mathrm{~h}$ after treatment. Fluorescence of Cy5-fluorophore in axons shows the uptake and trafficking of both tau monomers and oligomers.

phosphorylation levels of tau proteins at phospho-epitopes T231 (Fig. 3C) and S396/404 (Fig. 3D) were measured $7 \mathrm{~d}$ after tau seeding using high-content imaging. There was a significant increase in the percentage of cells with hyperphosphorylated tau (6.35 \pm 0.96 -fold increase in cells positive for phospho-epitope T231, $p<0.05 ; 2.14 \pm 0.18$-fold increase in cells positive for phospho-epitope S396/404, $p<0.05$ ) after treatment with tau oligomer seeds compared with vehicle-treated cells. Cells seeded with monomeric tau did not show any difference in hyperphosphorylated tau versus vehicle-treated cells (Fig. 3C,D).

To further investigate whether tau oligomers can induce the formation of insoluble aggregated species, we performed sarkosyl extraction of the hiPSC neurons treated with $50 \mathrm{~nm}$ of tau seeds. To ensure the detection of intracellular tau, we used the anti-phospho tau antibody (PHF1) in our Western blots, as recombinant tau seed preparations are not phosphorylated. Treatment with $50 \mathrm{~nm}$ did not show evident difference between the vehicle-treated, monomer-treated, and oligomertreated cells (data not shown). To test whether this lack of effect was due to the sensitivity of Western blot method versus high-content image analysis, which enables analysis at the single-cell level, we repeated the experiment with the addition of $200 \mathrm{~nm}$ tau seeds. Treatment with this higher concentration of tau oligomers caused significant increases $(p<0.05)$ in the levels of phospho-tau in sarkosyl-insoluble fraction, while the levels of phospho-tau in the soluble fraction was not increased compared with vehicle and monomer treatment. Tau monomer seeding did not affect the levels of phospho-tau in either the sarkosyl-soluble or the sarkosyl-insoluble fraction (Fig. $3 E)$. These data confirmed that tau oligomers can induce aggregation of insoluble, hyperphosphorylated tau species in hiPSC neurons. Numerous reports have demonstrated that autophagy is a major process involved in the clearance of intracellular tau aggregates in various in vitro and in vivo models of tau pathology (Berger et al., 2006; Frederick et al., 2015; Friedman et al., 2015). Rapamycin is a well known activator of autophagy (Berger et al., 2006). We examined the effect of rapamycin ( $100 \mathrm{~nm}$ ) by adding it to neurons $24 \mathrm{~h}$ before seeding with tau oligomers. Rapamycin pretreatment significantly decreased $(p<0.005)$ the percentage of cells positive for MC1 staining, suggesting that activation of autophagy was able to promote the clearance of the induced aggregated tau (Fig. $3 F$ ). To verify that rapamycin pretreatment activated autophagy, we performed immunostaining against LC3 protein, a reliable marker of autophagosomes (Kabeya et al., 2000; Mizushima, 2011; Klionsky et al., 2012). The number of intracellular LC3positive puncta measured by high-content image analysis was increased $(p<0.05)$ in cells treated with rapamycin compared with vehicle (DMSO), providing evidence for the induction of autophagy in the hiPSC neurons (Fig. 3G). 

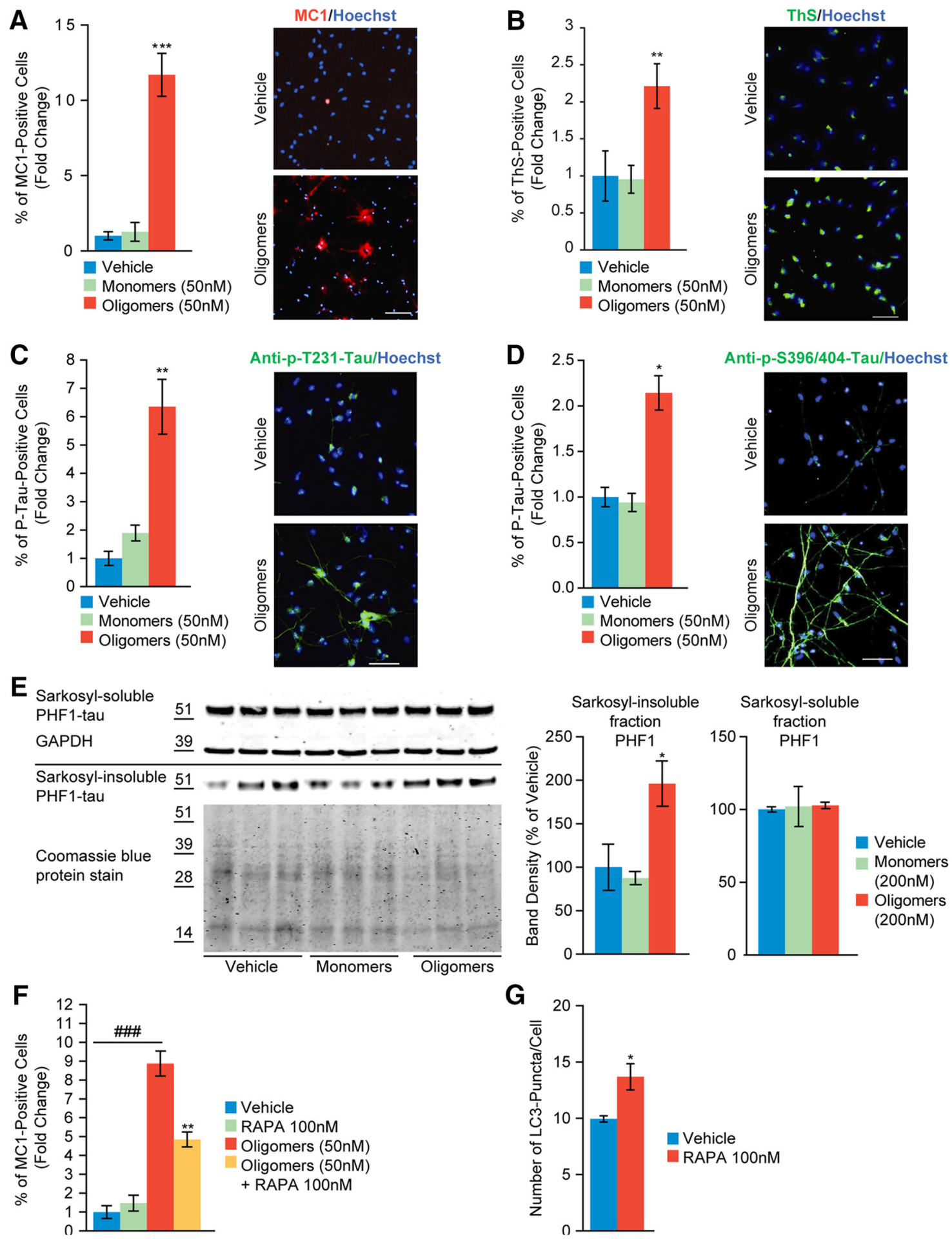

Figure 3. Tau oligomers induce accumulation of pathological tau. $\boldsymbol{A}-\boldsymbol{D}$, High-content image analyses of the hiPSC neurons $7 \mathrm{~d}$ after seeding with tau monomers and oligomers, and stained for pathological tau using MC1 antibody $(\boldsymbol{A})$, ThS staining $(\boldsymbol{B})$, and anti-phospho T231 tau $(\boldsymbol{C})$ and anti-phospho S396/404 tau antibody $(\boldsymbol{D})$. All graphs represent the percentage of cells positive for markers of pathological tau expressed as fold change versus vehicle. Representative images show cells positive for MC1 ( $\boldsymbol{A}$, red), ThS ( $\boldsymbol{B}$, green), and phospho-tau staining ( $\boldsymbol{C}, \boldsymbol{D}$, green). Hoechst was used to stain nuclei (blue). Scale bar, $50 \mu \mathrm{m}\left(n=6\right.$ wells per condition, 9 fields, ANOVA with post hoc Dunnett's test vs vehicle, ${ }^{*} p<0.05$, ${ }^{* *} p<$ $0.005,{ }^{* *} p<0.0001$ ). $\boldsymbol{E}$, Images of Western blot analysis of sarkosyl-soluble and sarkosyl-insoluble fractions. PHF1 antibody (anti-phospho S396/404) was used to detected phospho-tau; GAPDH and Coomassie blue protein stain were used as a loading control. Molecular weight in kilodaltons is indicated along the blot. Western blots were quantified by densitometric analysis (graphs; $n=3$, ANOVA with post hoc Dunnett's test vs vehicle; insoluble fraction, ${ }^{*} p<0.05$; soluble fraction, $p=0.19$ ). $\boldsymbol{F}$, Graph represents the percentage of cells positive for MC1 staining $7 \mathrm{~d}$ after seeding after treatment with rapamycin. Rapamycin was added $24 \mathrm{~h}$ before tau seeding. DMSO was used as a vehicle ( $n=6$ wells per condition, 9 fields, ANOVA with post hoc Dunnett's test, ${ }^{\# \# \#} p<0.0001$ vs vehicle ${ }^{* *} p<0.005$ vs oligomer). G, Graph shows the number of LC3-positive autophagosomes per cell after rapamycin pretreatment ( $n=3$ wells per condition, 9 fields, Student's $t$ test, ${ }^{*} p<0.05$ ). All values are expressed as mean \pm SEM. 
A

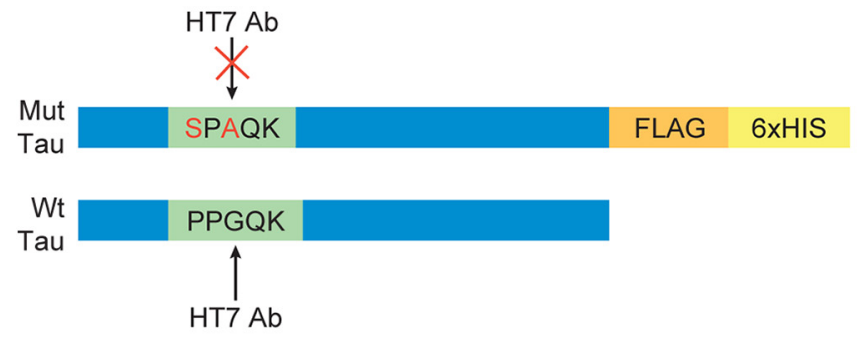

B
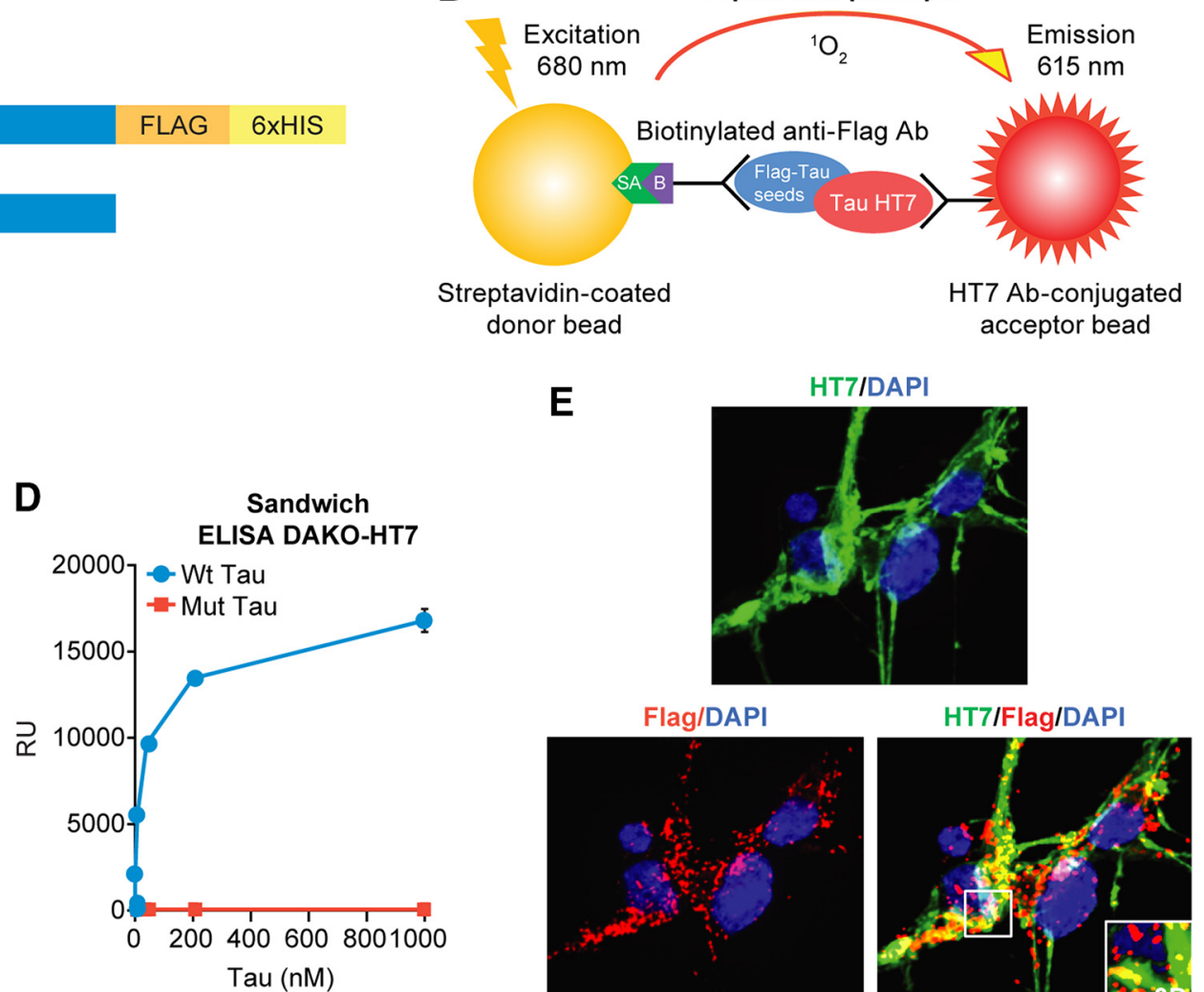

D
E
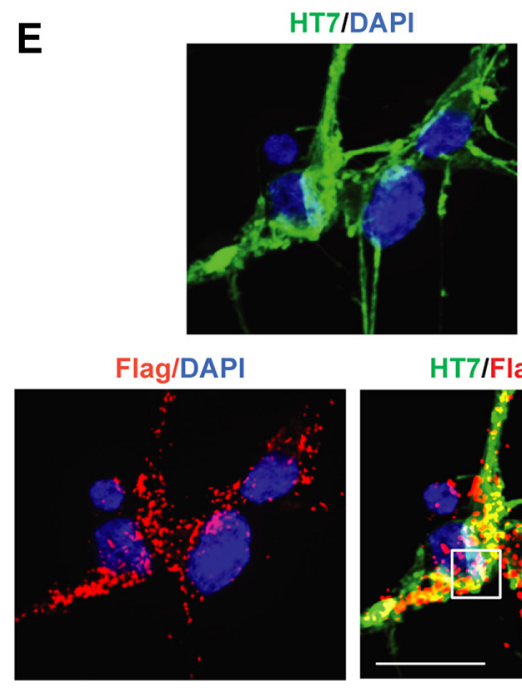

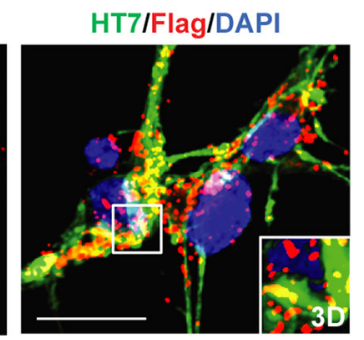

Figure 4. Exogenous tau oligomers recruit endogenous tau protein. $\mathbf{A}$, Illustration of tau protein tagged with Flag-6xHis and mutated at the binding site of the HT7 antibody. $\boldsymbol{B}$, Illustration of AlphaLISA assay principle; interaction between exogenous (Flag detected) and endogenous (HT7 detected) tau proteins brings acceptor and donor beads in very close proximity, which enables energy transfer from one bead to another, generating luminescence signal. C, Graph represents the Flag-HT7 AlphaLISA luminescence signal (fold change vs vehicle) from hiPSC-neuronal lysates seeded with two concentrations ( 50 and $250 \mathrm{~nm}$ ) of tau monomers and oligomers. Lysates were harvested $4 \mathrm{~d}$ after seeding $\left(n=3\right.$ per condition, ANOVA with post hoc Dunnett's test, ${ }^{*} p<0.05 \mathrm{vs}$ vehicle, ${ }^{* *} p<0.005$ vs vehicle). D, Graph shows sandwich ELISA signal with HT7 and total tau antibody used to detect HT7-mutant tau and wild-type (wt) tau. RU, Relative units. All values are expressed as mean \pm SEM. $\boldsymbol{E}$, High-resolution confocal images show the colocalization of Flag (exogenous tau) and HT7 (endogenous tau) staining in hiPSC neurons $4 \mathrm{~d}$ after treatment with tau-Flag oligomers. Higher magnification shows three-dimensional reconstruction of the Z-stacked images processed with Volocity program. Scale bar, $50 \mu \mathrm{m}$.

\section{Tau oligomer seeds recruited endogenous tau protein}

To differentiate endogenous tau from seeded tau oligomers, we mutated the HT7 antibody recognition site of our tau recombinant protein and conjugated it with Flag-6xHis (Fig. 4A). We used AlphaLISA technology to detect the formation of protein aggregates. An increase in HT7-Flag AlphaLISA signal revealed the close proximity between endogenous tau (labeled with HT7 antibody conjugated to acceptor beads) and tau seeds (labeled with biotinylated anti-Flag antibody and streptavidin-coated donor beads; Fig. 4B). The addition of 50 or $250 \mathrm{~nm}$ tau oligomer seeds to the hiPSC neurons for $4 \mathrm{~d}$ resulted in a significant increase $(p<0.05)$ in AlphaLISA signal compared with vehicletreated neurons (Fig. $4 C$ ). No change was observed when cells were treated with tau monomer seeds compared with vehicle treatment (Fig. 4C). In addition, a sandwich ELISA using total tau antibody (Dako) as a capture antibody and HT7 antibody for detection showed a complete loss of signal from the HT7 mutant tau oligomer seeds compared with the wild-type tau oligomer seeds, confirming the mutation of the HT7 antibody recognition site (Fig. 4D). High-resolution confocal microscopy showed colocalization of Flag and HT7 staining, providing additional evidence that tau-Flag-6xHis seeds are interacting with endogenous tau to promote further pathological assembly (Fig. 4E).

Together, these data suggest that tau oligomers are internalized and are able to seed hiPSC neurons recruiting endogenous tau protein to induce pathological changes and aggregation.
Tau oligomer seeds caused neurite degeneration and neuronal loss

To investigate the outcomes of tau oligomer seed-induced intracellular tau aggregation on neuronal survival, we determined the effect of increasing concentration of tau oligomers on MC1 staining, neurite outgrowth, and neuronal count $7 \mathrm{~d}$ after seeding in hiPSC neurons. Neurite outgrowth of all neuronal processes was used as an indicator of neurite degeneration. Neurite outgrowth was measured using high-content image analysis software (Harmony 3.1.1, PerkinElmer), which detects neurites extending from a cell body based on immunofluorescence. A representative image demonstrating the detection of the neurites and cells using this algorithm is shown in Figure $5 A$. Neurite outgrowth was quantified by measuring total neurite length per cell and number of segments per cell (neurites between two intersecting points), averaged per well. The analyses showed a concentrationdependent increase in the number of MC1-positive neurons starting at $50 \mathrm{~nm}$ tau oligomeric seeds (Fig. 5B). This increase in pathological tau conformation was associated with a significant reduction in neurite length and segments (based on total tau immunostaining; Fig. 5C), as well as a decrease in the number of healthy nuclei stained with Hoechst dye (Fig. 5D). At $50 \mathrm{~nm}$ tau oligomer treatment, $8.3 \pm 0.8 \%(p<0.0001)$ of the cells were MC1 positive. Compared with vehicle, there was a $27.4 \pm 2.4 \%$ $(p<0.0001)$ reduction in neurite length and $12.9 \pm 3.7 \%$ reduction in the number of healthy nuclei. The highest concentration 
A

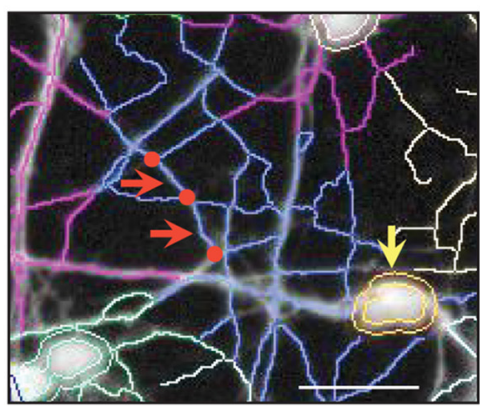

C

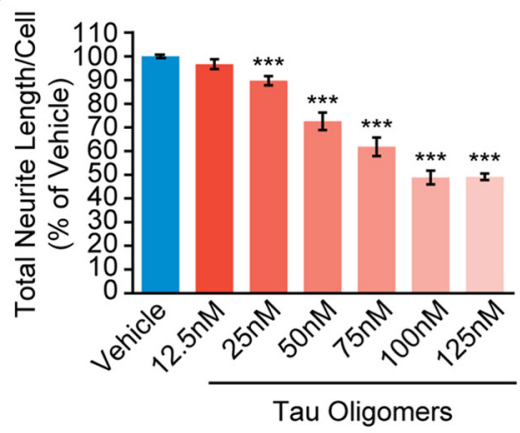

B
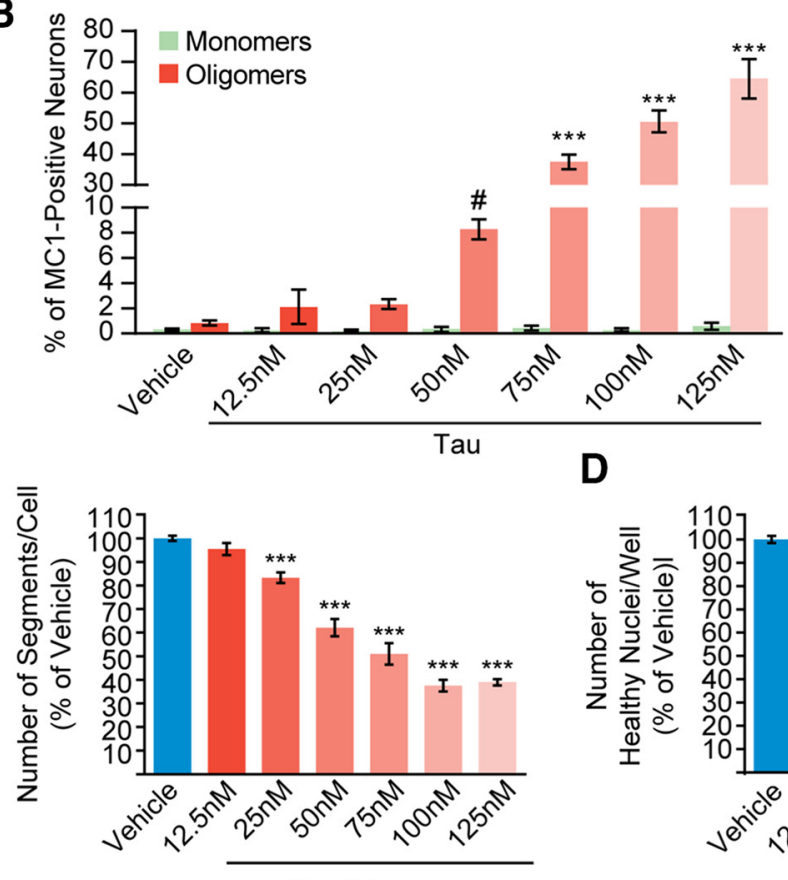

Tau Oligomers

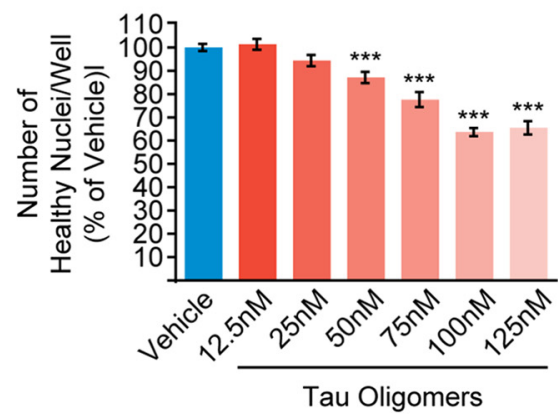

$\mathbf{F}$

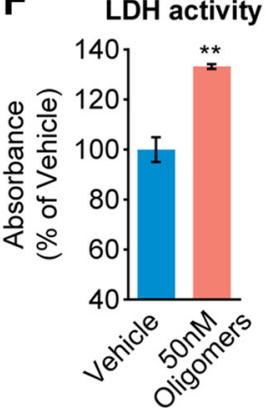

G
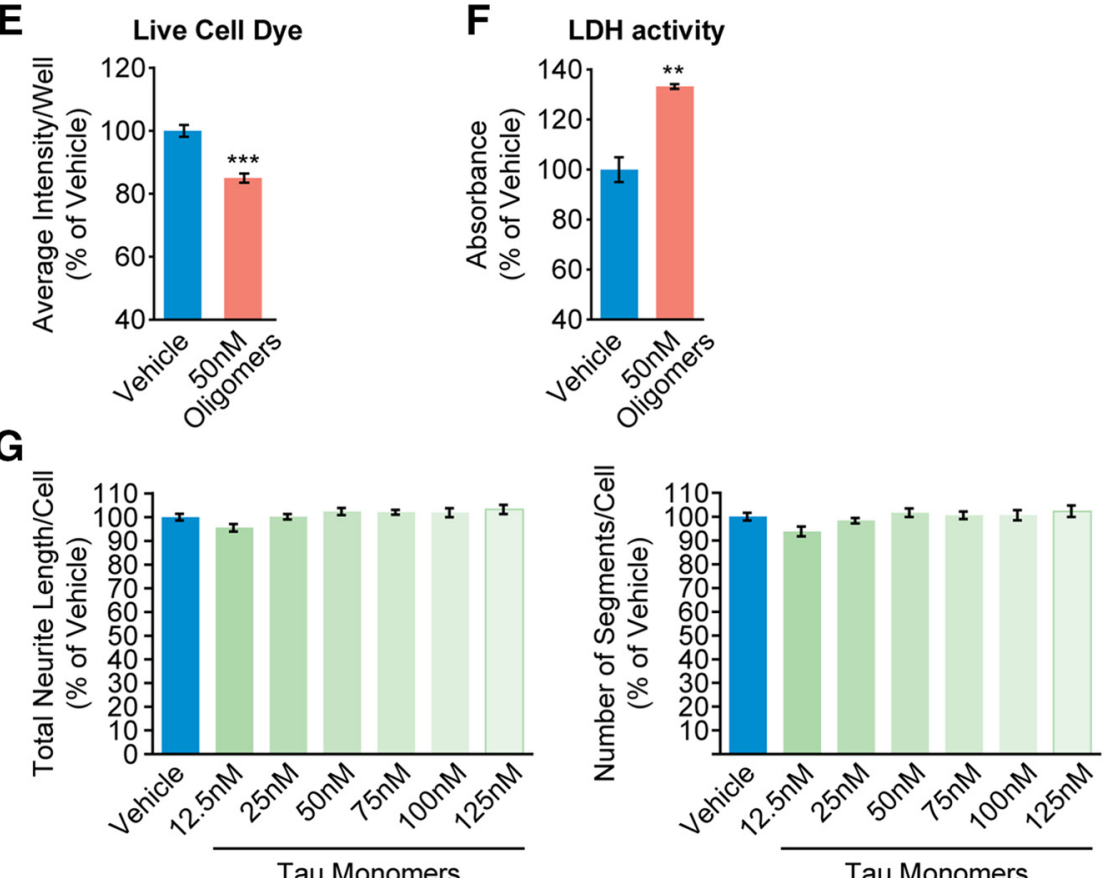

H

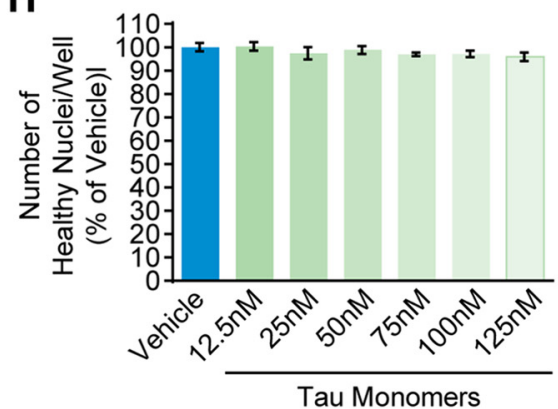

Figure 5. Tau oligomers cause neurite retraction and neurotoxicity. $A$, Image demonstrates the masks of enlarged nuclei, representing cell body (yellow arrow) and neurites that extend from the cell body, detected by Harmony 3.1.1 (PerkinElmer) image-analysis software, based on fluorescence from Hoechst dye and tau immunostaining. Neurite segments (red arrows) are calculated as parts of the neurites between neurite intersections (red dots). B, Quantification of percentage of neurons MC1-positive for pathological tau $7 \mathrm{~d}$ after the treatment with increased concentrations of tau monomers or oligomers. Oligomer treatment resulted in concentration-dependent increase in MC1-positive cells, compared with monomer treatment that showed no effect, only the background of the MC1 staining ( $<0.5 \%$ MC1-positive cells; $n=6$ wells per condition, 9 fields, for oligomers ANOVA with post hoc Dunnett's test, ${ }^{* * *} p<0.0001$ vs vehicle, Student's $t$ test, ${ }^{\# \# \# ~} p<0.0001$ vs vehicle, for monomers, $p=0.93)$. C, Graphs represent high-content image analyses of neurite outgrowth $7 \mathrm{~d}$ after seeding with different concentrations of tau oligomers. The graph on the left shows tau oligomers' dose-dependent decrease of total neurite length per cell, while the graph on the right shows number of neurite segments per cell. Neurites were detected using total tau staining. $\boldsymbol{D}$, Graph shows the effect of increasing concentration of tau oligomers on the number of healthy nuclei (detected with Hoechst dye) $7 \mathrm{~d}$ after seeding ( $C$ and D: $n=6$ wells per condition, 9 fields, ANOVA with post hoc Dunnett's test vs vehicle, $\left.{ }^{* * *} p<0.0001\right)$. E, High-content image analyses of the intensity of live-cell dye $7 \mathrm{~d}$ after seeding with $50 \mathrm{~nm}$ tau oligomers. Average fluorescence intensity of cells per well was expressed in graph as a percentage of vehicle-treated cells ( $n=6$ wells per condition, 9 fields, Student's t test, ${ }^{* * *} p<0.0001$ vs vehicle). $F$, LDH activity detected in the medium of cells treated with tau oligomers $\left(50 \mathrm{~nm}\right.$ ) and vehicle-treated cells ( $n=3$ per condition, Student's $t$ test, $\left.{ }^{* *} p<0.005\right)$. G, Graphs present high-content image analyses of neurite outgrowth $7 \mathrm{~d}$ after seeding with different concentrations of tau monomers. $\boldsymbol{H}$, Graph shows the number of healthy nuclei (detected with Hoechst dye) $7 \mathrm{~d}$ after treatment with increasing concentration of tau monomers ( $G$ and $\boldsymbol{H}: n=6$ wells per condition, 9 fields, ANOVA with post hoc Dunnett's test vs vehicle, no significant change). All values are expressed as mean \pm SEM. 
A

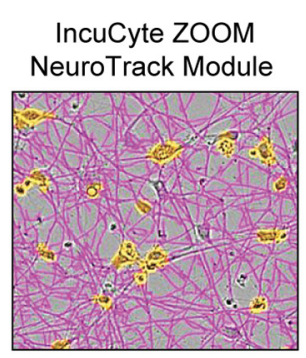

Neurite Mask

Cell-Body Mask
B

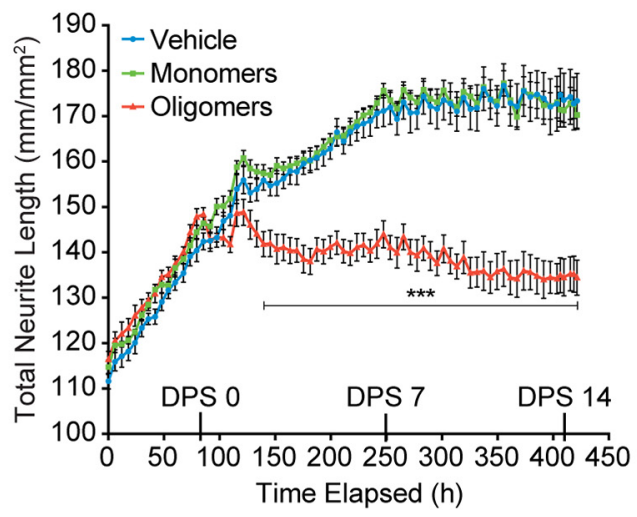

D

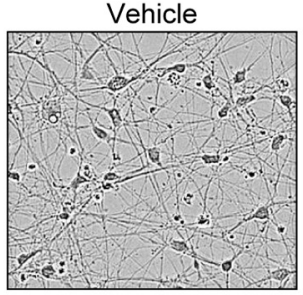

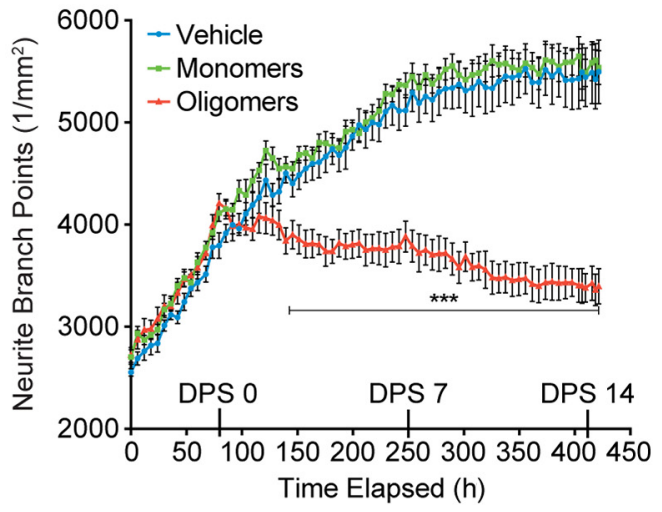

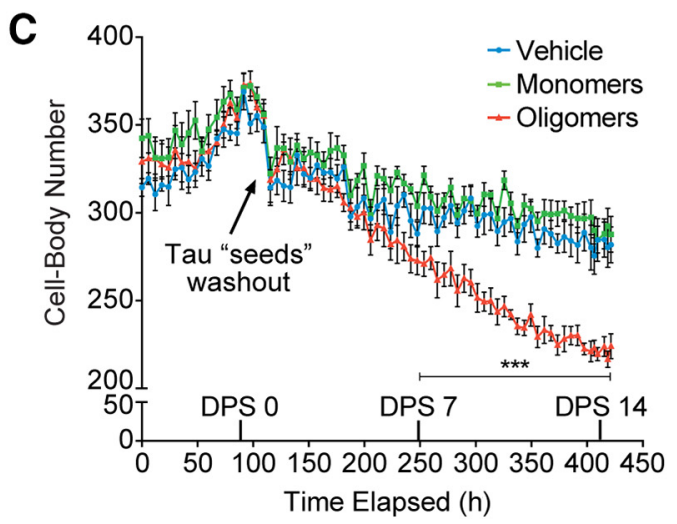

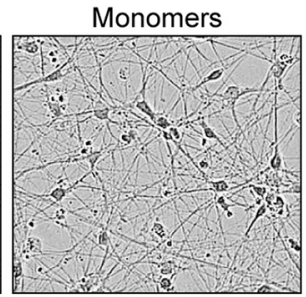

Day Post-seeding 7

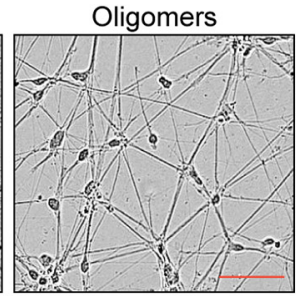

Oligomers

Figure 6. Time-lapse imaging revealed progressive neurite degeneration in neurons treated with tau oligomers. $\boldsymbol{A}$, Representative image illustrates the definition marks of neurites (pink) and cell bodies (yellow) defined with NeuroTrack algorithm based on phase contrast. $\boldsymbol{B}$, Graphs show the total neurite length (left) and number of branching points (right) per square millimeter, averaged from four imaged fields. Results were obtained from time-lapse live-cell imaging of the neurons treated with $50 \mathrm{~nm}$ tau seeds. DPS, Days postseeding. C, Graph presents the long-term quantification of the number of cell bodies in neuronal cultures treated with tau seeds. Arrow indicates plate removal from the imaging system for the treatment and washout of tau seeds (all graphs: $n=6$ wells per condition, 4 fields, ANOVA with post hoc Dunnett's test vs vehicle, ${ }^{* * *} p<0.0001$ ). All values are expressed as mean \pm SEM. $D$, Bright-field images acquired with IncuCyte Z00M demonstrating the reduced neurite network of the hiPSC neurons $7 \mathrm{~d}$ after seeding with tau oligomers. Scale bar, $50 \mu \mathrm{m}$.

of tau oligomers tested at $125 \mathrm{~nm}$ resulted in $64.5 \pm 6.3 \%(p<$ 0.0001 ) of the cells positive for $\mathrm{MC1}$, and further reduced neurite outgrowth and the percentage of healthy nuclei, lowering them by $50.9 \pm 1.3 \%(p<0.0001)$ and $34.5 \pm 2.9 \%(p<0.0001)$, respectively. The effect of tau oligomer seeds on neuronal viability was further confirmed using a live-cell dye, calcein AM, and the measure of LDH activity, an indicator of cell death, in the cell medium. At the concentration of $50 \mathrm{~nm}$ tau oligomers, $7 \mathrm{~d}$ after seeding, hiPSC neurons exhibited a $25.2 \pm 1.9 \%(p<0.0001)$ decrease in the intensity of calcein $\mathrm{AM}$ and a $33.2 \pm 0.9 \%(p<$ 0.005 ) increase in LDH activity compared with vehicle (Fig. $5 E, F)$.

In addition, increasing concentrations of tau monomers had no effect on MC1 staining, neurite outgrowth, and neuronal count $7 \mathrm{~d}$ after seeding in hiPSC neurons (Fig. $5 B, G, H$ ).

Together, these data imply that tau oligomers, but not tau monomers, are neurotoxic for hiPSC neurons, setting the basis for the neurodegenerative process that causes neurite retraction and, ultimately, cell death.

\section{Tau oligomers induced long-term progressive degeneration of} neurites accompanied with neuronal death

The study of the long-term effect of tau oligomers on neurite morphology and viability was performed using continuous, 3 week live-cell imaging. The NeuroTrack module was used to measure neuronal processes and number of cell bodies of live cells based on phase-contrast imaging. Representative image of detection masks of the NeuroTrack module is shown in Figure
$6 A$. These analyses revealed that neurons treated with $50 \mathrm{~nm}$ tau oligomers exhibited progressive neurite degeneration determined by the reduction in both neurite length and branching over the course of 2 weeks after tau seeding (Fig. 6B). In contrast, vehicle-treated and monomer-treated neurons showed a continuous increase in neurite growth and branching. From the day of treatment (day postseeding 0 ) until the last time point (day postseeding 14) both vehicle-treated and monomer-treated neurons exhibited significant increases in neurite length of $32.96 \pm 6.54$ $\mathrm{mm} / \mathrm{mm}^{2}(p<0.005)$ and $25.82 \pm 3.46 \mathrm{~mm} / \mathrm{mm}^{2}(p<0.0001)$, respectively, suggesting a dynamic growth of neurites. The oligomer-treated cells had a significant decrease of $13.40 \pm 4.27$ $\mathrm{mm} / \mathrm{mm}^{2}(p<0.05)$ in neurite length, suggesting neurite retraction during this 2 week period. Of note, the three treatment groups started with a similar neurite outgrowth profile from the beginning of the imaging until the time of treatment (day 0 ). In addition, the quantification of the cell bodies revealed a timedependent decrease in cell number in the three treatment groups, indicative of a progressive loss of human neurons during the 3 weeks of culturing. Importantly, greater cell loss was observed in the tau oligomer-seeded neurons $(29.71 \pm 7.07 \%, p<0.0001)$ compared with vehicle-treated neurons $(10.33 \pm 10.23 \%)$, whereas tau monomer-seeded neurons $(10.74 \pm 10.17 \%)$ did not show any difference from vehicle-treated neurons (Fig. 6B). Acute differences in cell number between day 0 and 1 after seeding are a treatment artifact induced by removal of the plate from the imaging system and extensive wash steps (Fig. $6 \mathrm{C}$, arrow on graph). The percentage change of cell number was then calcu- 
lated based on the cell number after the wash. Interestingly, in oligomer-treated neurons, the significant loss of cell bodies compared with a vehicle-treated and monomer-treated groups, was detected $7 \mathrm{~d}$ after seeding (Fig. $6 C$ ), while modest, though significant, neurite degeneration started at the earlier time points (Fig. $6 B)$. This observation further supports the importance of neurite outgrowth as an indication of neurodegeneration that precedes the cell death. Together, these data indicate that treatment with tau oligomers causes long-term neurite degeneration and neuronal loss.

\section{hiPSC neurons affected with pathological tau aggregates exhibited neuronal death and neurite degeneration}

We next investigated the relationship between the induction of tau pathology and the neurodegenerative processes. For this analysis, hiPSC neurons were seeded with $50 \mathrm{~nm}$ tau oligomers or monomers, and neurite outgrowth was measured at $0,7,14$, and $21 \mathrm{~d}$ after seeding. Consistent with the previous results of live-cell imaging, these analyses of neurite outgrowth based on total tau immunostaining showed a time-dependent decrease in neurite length and number of segments of neurons treated with tau oligomers, compared with vehicle-treated neurons at all time points (Fig. 7A). In contrast, there was no difference between monomerseeded and vehicle-treated cells (Fig. 7A). To confirm induction of neurodegeneration, we measured neurite outgrowth using immunostaining with two neuronal-specific proteins, MAP2 and TUJ-1. Fourteen days after oligomer seeding, neurons showed a significant reduction in length and branching of TUJ-1-labeled $(p<0.0001, p<0.005)$ or MAP2-labeled $(p<0.05)$ neurites compared with vehicle-treated cells (Fig. $7 B, C$ ), consistent with the previous results obtained using tau staining. Together, these results demonstrate the toxic effect of tau oligomer seeds on the neurite network. In parallel, we performed double staining to detect neurites, using tau or MAP2, and tau aggregates, using MC1. Based on the MC1 labeling, we were able to differentiate neurons with significant tau aggregates and to measure neurite outgrowth in MC1-positive population of neurons. Fourteen days after tau oligomer seeding, we observed a significant reduction in neurite length in the MC1-positive cells compared with non-MC1 cells (Fig. 7D,E). The neurite outgrowth of the non-MC1 tau oligomer-treated cells was similar to that of the vehicle-treated cells (Fig. $7 D, E$ ). For example, in the tau oligomer-treated neurons, the length of neurites of non-MC1 cells, based on tau staining, was $586.59 \pm 15.70 \mu \mathrm{m}$, whereas in MC1-positive cells, the length was significantly shorter (388.39 \pm $56.99 \mu \mathrm{m}, p<0.005$; Fig. $7 D$ ). Neurite length of the non-MC1 tau oligomer-treated cells did not significantly differ from that of vehicle-treated cells $(642.89 \pm 18.60 \mu \mathrm{m}$; Fig. $7 D)$. In addition, high-resolution confocal images showed the localization of MC1positive pathological tau in both neuritic and somatoneuritic cell compartments. These specific areas of $\mathrm{MC} 1$ accumulation are in agreement with the effect of pathological tau on neurite degeneration (Fig. $7 D, E)$.

In addition, consistent with the results of the live-cell analyses (Fig. 6), quantification of the cell nuclei stained with Hoechst revealed a time-dependent decrease in cell number in the three groups of treatment, reflecting a progressive loss of human neurons over the 4 weeks of culture. The reduction of cell viability was significantly higher $(p<0.005)$ in the tau oligomer-seeded neurons compared with vehicle-treated neurons at the three time points, whereas the tau monomer-seeded neurons did not show any difference from the vehicle-treated cells (Fig. $7 F$ ). In this experiment, we also detected the formation of tau aggregates us- ing MC1 staining in tau oligomer-seeded neurons. We were able to differentiate the change in viable cell number between $\mathrm{MC1}$ positive and non-MC1 cells at different time points after seeding. We found a significant decrease $(p<0.0001)$ in cell number from the MC1-positive neuronal population 14 and $21 \mathrm{~d}$ after seeding compared with the $7 \mathrm{~d}$ time point, whereas the number of non$\mathrm{MC1}$ cells was unchanged (no MC1 pathological tau was observed at day 0 of seeding). These data provided evidence of a progressive neuronal death affecting specifically the neuronal population harboring pathological tau aggregates (MC1-positive neurons; Fig. $7 G$ ). These results provide evidence that pathological tau aggregation induced by exogenous tau oligomers contribute to neurodegenerative processes and lead to neuronal death.

\section{Tau oligomers disrupted synaptic integrity and intracellular calcium levels}

Having observed the ability of tau oligomer seeds to induce neurodegeneration, we next explored the functional consequences of tau oligomers on synaptic integrity and neuronal activity. To measure synaptic changes, we performed high-content quantification of the puncta positive for synaptic markers synapsin I and synaptophysin. Synapsin 1 and synaptophysin are well characterized markers used for immunostaining of synaptic contacts (Fletcher et al., 1991). Fourteen days after seeding with tau oligomers, hiPSC neurons showed a significant decrease $(p<0.05)$ in synaptic puncta compared with vehicle-treated neurons (Fig. $8 A$ ). These iCell hiPSC neurons were characterized as predominantly GABAergic and glutamatergic (Haythornthwaite et al., 2012; Xu et al., 2013). Therefore, neuronal activity was assessed by determining the levels of GABA and glutamate released in the cell media. In neuronal cultures seeded with tau oligomers, we measured a moderate but significant increase $(p<0.05)$ in basal GABA release, whereas glutamate release was unaffected (Fig. $8 B)$. This result suggests that tau oligomer seeds may preferentially affect the function of GABAergic hiPSC neurons and inhibitory neurotransmitter signaling.

Neuronal calcium signaling plays a crucial role in controlling the release of neurotransmitters, membrane excitability, and overall neuronal activity (Bezprozvanny and Mattson, 2008; Supnet and Bezprozvanny, 2010). High-content imaging was used to monitor intracellular $\mathrm{Ca}^{2+}$ at single-cell level in hiPSC neuronal culture loaded with calcium-sensitive fluorescent dye. Neuronal stimulation with $30 \mu \mathrm{M}$ NMDA, used as a control for a $\mathrm{Ca}^{2+}$ dye, resulted in an increased fluorescence as represented by Figure $8 C$. Fourteen days after seeding, intensity of $\mathrm{Ca}^{2+}$ fluorescence in hiPSC neurons seeded with tau oligomers was significantly higher $(755 \pm 24.24$ fluorescence units, $p<0.0001)$ than the levels in vehicle-treated neurons ( $536 \pm 33.43$ fluorescence units; Fig. $8 C$ ). These results suggest that tau oligomers induce alterations in neuronal activity. Interestingly, in neurons seeded with tau oligomers, the impairments in synaptic integrity, neurotransmitter release, and calcium signaling occurred concurrently with the tau aggregate-induced deficit in neurite outgrowth, thereby correlating structural abnormalities with functional deficiency.

\section{Discussion}

It is now evident that exogenously applied aggregated tau can induce tau pathology in cellular and animal models. However, it is still unknown which of the various tau multimeric misfolded species are most efficient in triggering aggregation and spreading of tau pathology in AD. In the majority of studies, tau seeds were prepared from either recombinant mutant tau (Guo and Lee, 2011; Iba et al., 2013) or from brain extracts of tau mutant trans- 

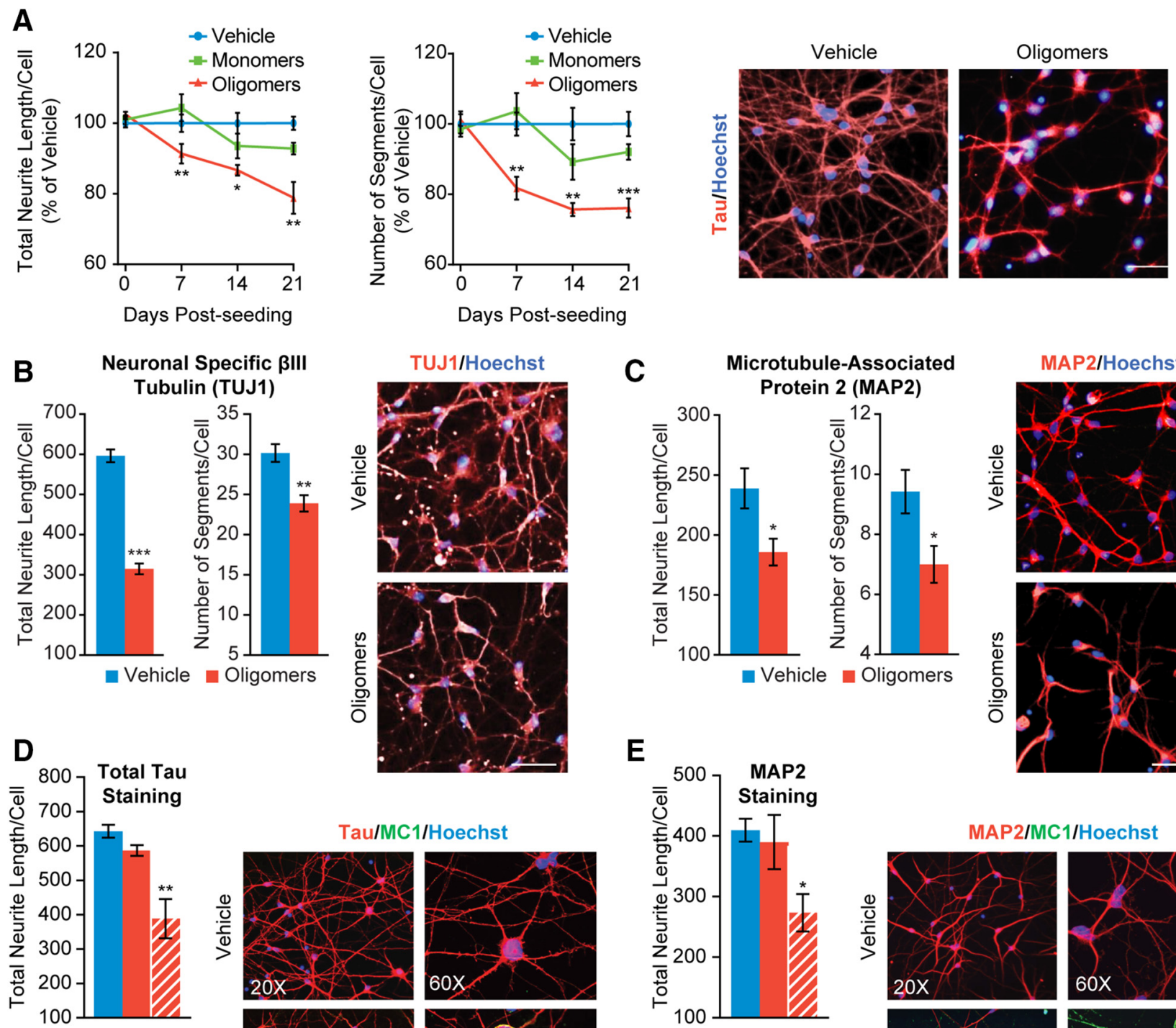

C

Microtubule-Associated
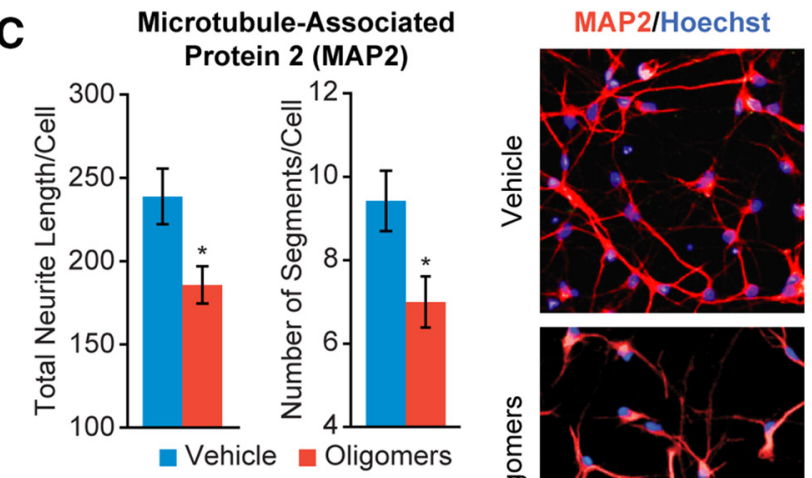

E
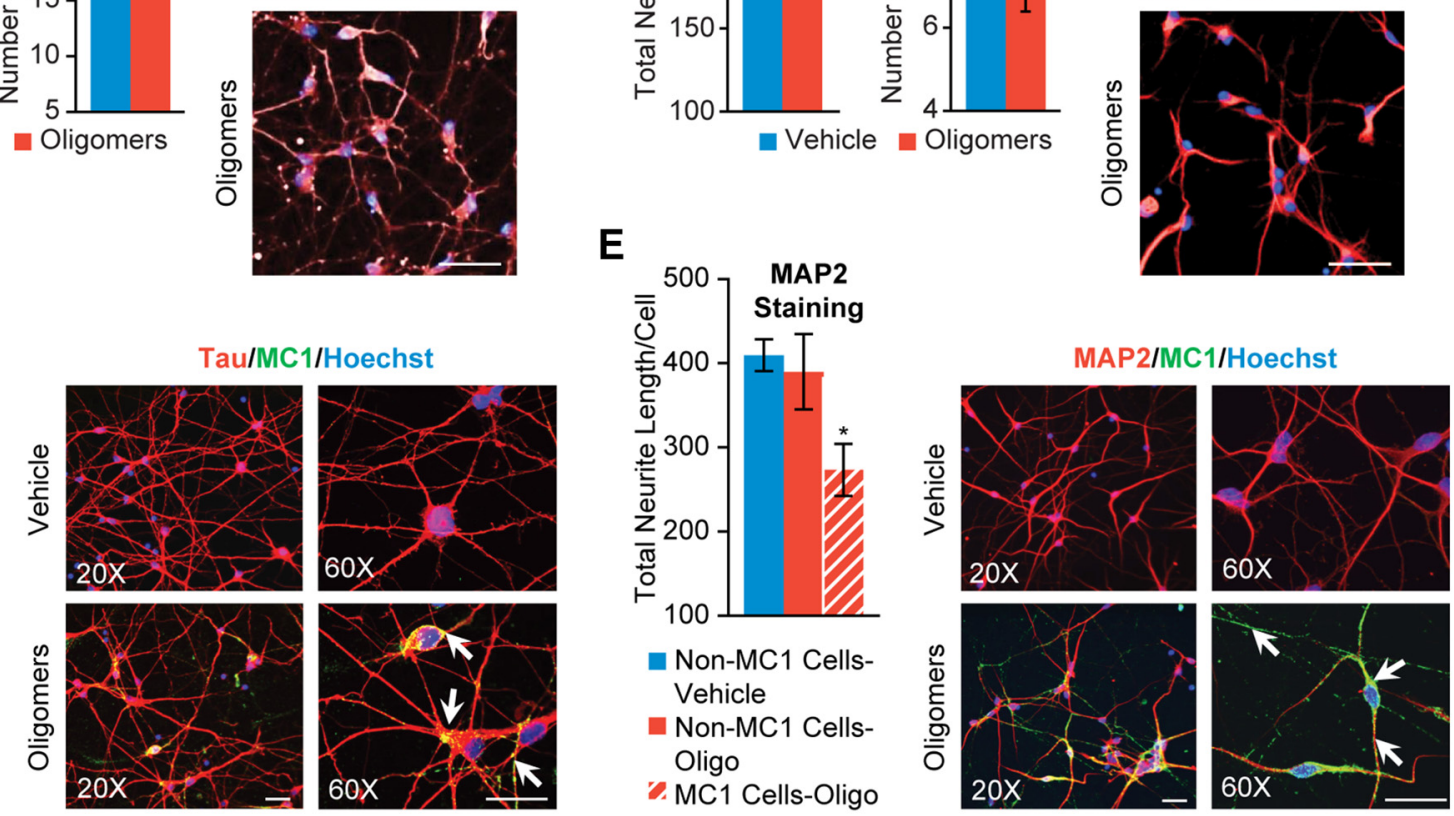

- Non-MC1 Cells-

Vehicle

Non-MC1 Cells-

Oligo

2 MC1 Cells-Oligo
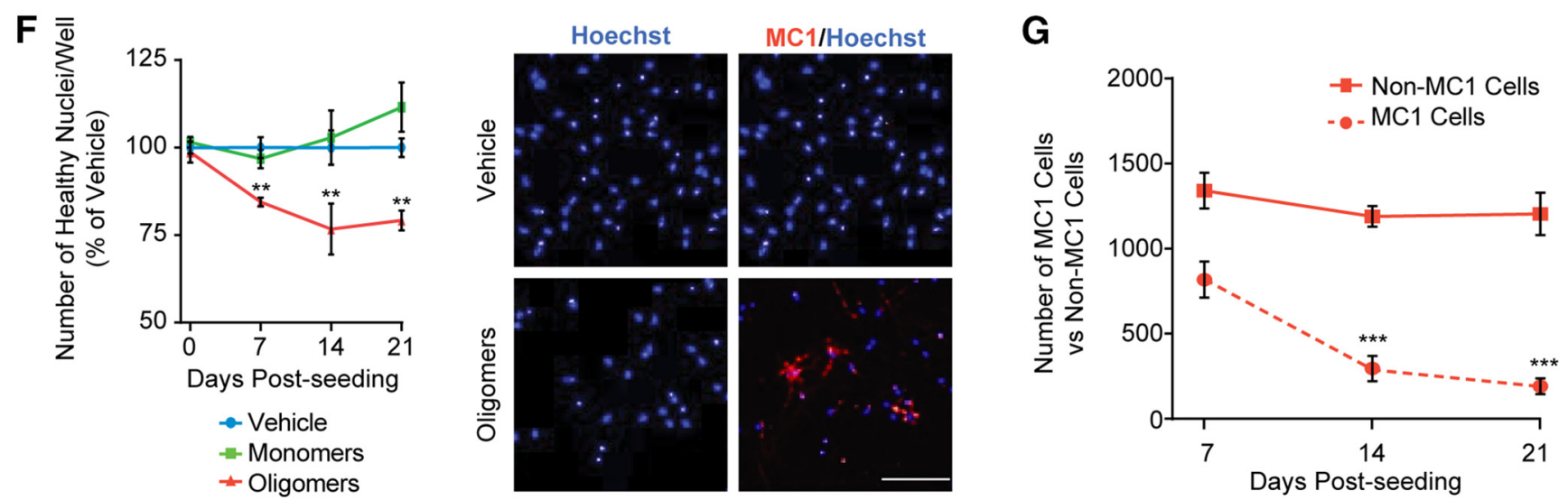

Figure 7. Accumulation of pathological tau causes neuronal degeneration. A, High-content image analyses of neurite outgrowth expressed in graphs as total neurite length (in micrometers) per cell (left) and number of segments per cell (right), during a time course of 3 weeks after seeding with $50 \mathrm{~nm}$ tau oligomers, monomers, and vehicle-treated hiPSC neurons. Results are expressed as a percentage change from the vehicle within each time point. Representative images (right) demonstrate the neurite network reduction of hiPSC neurons treated with tau oligomers compared with vehicle-treated cells $14 \mathrm{~d}$ after seeding. Neurites were detected with total tau immunostaining (red). Hoechst was used to stain nuclei (blue). B, C, Graphs represent (Figure legend continues.) 
A
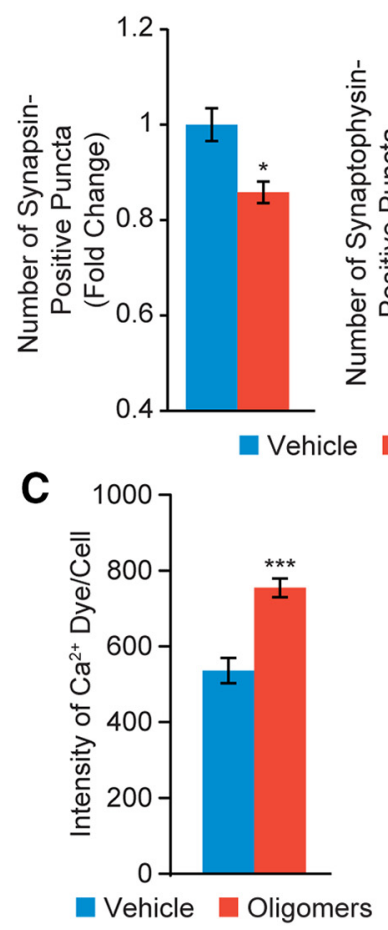

Synapsin/

Hoechst

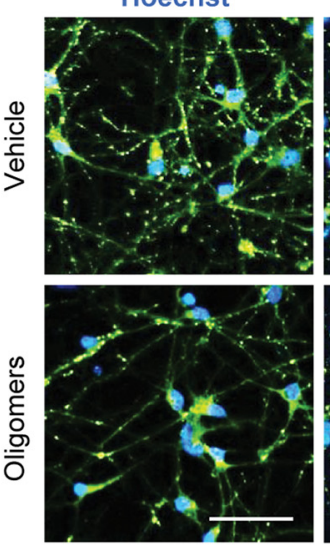

Synaptophysin/ Hoechst

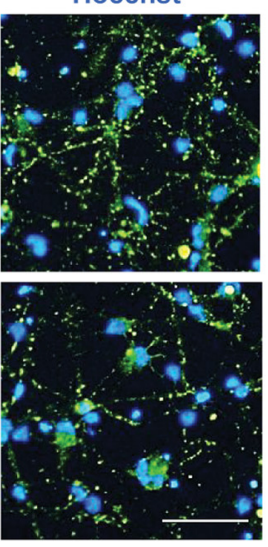

B

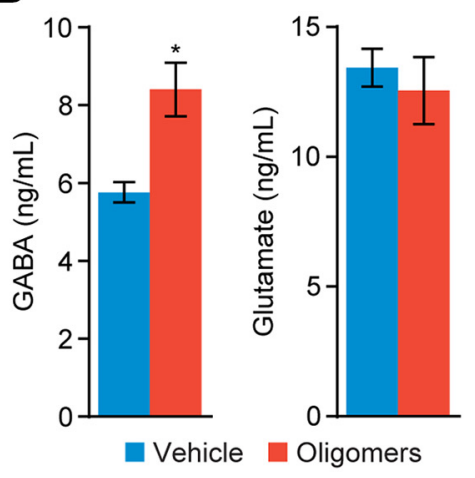

Figure 8. Pathogenic tau oligomers cause loss of synapses and disrupt intracellular calcium levels and neurotransmitter release. $A$, Graphs represent the quantification of number of puncta positive for the synaptic markers synapsin I and synaptophysin in hiPSC neurons $14 \mathrm{~d}$ after treatment with tau oligomers $\left(n=6\right.$ wells per condition, 9 fields, Student's $t$ test, $\left.{ }^{*} p<0.05\right)$. Representative images (to the right of graphs) of synapsin and synaptophysin staining (green) and Hoechst nuclear stain (blue). $\boldsymbol{B}$, Graphs show the levels of released neurotransmitter GABA and glutamate $14 \mathrm{~d}$ after tau oligomer seeding $\left(n=6\right.$ wells per condition, Student's $t$ test, $\left.{ }^{*} p<0.05\right)$. C, High-content image analyses of the $\mathrm{Ca}^{2+}$-dye intensity per cell $14 \mathrm{~d}$ after tau oligomer seeding ( $n=5$ wells per condition, 9 fields, Student's t test, ${ }^{* * *} p<0.0001$ ). Images show an increased intracellular fluorescence intensity of $\mathrm{Ca}^{2+}$ dye (green) in cells treated with tau oligomers compared with vehicle-treated cells. Treatment with $30 \mu \mathrm{M}$ NMDA was used to validate the increase of intracellular $\mathrm{Ca}^{2+}$ dye after neuronal activation. Nuclei were stained with Hoechst (blue). Scale bar, $50 \mu \mathrm{m}$.

genic mice (Clavaguera et al., 2009). In some cases, the in vitro and in vivo models also overexpressed mutant or wild-type tau (Clavaguera et al., 2009, 2013; Guo and Lee, 2011), rendering these systems even more susceptible to endogenous tau aggregation. However, there are no reported MAPT (microtubuleassociated protein tau) mutations in $\mathrm{AD}$ or evidence of increased

$\leftarrow$

(Figure legend continued.) the reduction in neurite length and reduction in number of segments of neurons treated with tau oligomers $14 \mathrm{~d}$ after seeding, when neurites were detected with TUJ1 ( $\boldsymbol{B}$ ) and MAP2 immunostaining ( $\boldsymbol{C} ; n=6$ wells per condition, 9 fields, Student's $t$ test, ${ }^{*} p<0.05,{ }^{* *} p<0.005,{ }^{* * *} p<0.0001$ vs vehicle). Representative images of neurite network detected with TUJ1 ( $\boldsymbol{B}$, red) and MAP2 ( $\boldsymbol{C}$, red) immunostaining. Nuclei were detected with Hoechst stain (blue). $\boldsymbol{D}, \boldsymbol{E}$, Graphs present high-content image analyses of total neurite length of MC1-positive and non-MC1-positive cells $14 \mathrm{~d}$ after treatment with tau oligomers and vehicle, when neurites were detected with tau $(\boldsymbol{D})$ and MAP2 $(\boldsymbol{E})$ immunostaining. Representative images of MC1/tau ( $\boldsymbol{D}$, right) and MC1/MAP2 ( $\boldsymbol{E}$, right) double staining that demonstrate the reduction of neurite network (red) in neuronal population positive for MC1 staining (green) $14 \mathrm{~d}$ after tau oligomer seeding. Higher-magnification images $(60 \times)$ show the localization of MC1 pathological tau on neuronal processes and somas, and also on the degenerating neurites (arrows). Nuclei were detected with Hoechst stain (blue). Scale bar, $50 \mu \mathrm{m}$. F, Graph shows the percentage of the number of healthy nuclei stained with Hoechst in neuronal cultures treated with tau seeds compared with vehicle at indicated time points. Representative images of MC1 (red) and Hoechst nuclear stain (blue) $14 \mathrm{~d}$ after treatment with tau oligomers showing a reduction in the number of Hoechst-positive nuclei in tau oligomer-treated neurons compared with vehicle. Scale bar, $100 \mu \mathrm{m}(A, D-F: n=6$ wells per condition, 9 fields, ANOVA with post hoc Dunnett's test vs vehicle, ${ }^{*} p<0.05,{ }^{* *} p<0.005$, $\left.{ }^{* * *} p<0.0001\right)$. G, Number of MC1positive cells compared with number of non-MC1 cells during a time course of 3 weeks after seeding with $50 \mathrm{~nm}$ tau oligomers ( ${ }^{* * *} p<0.0001$ vs $7 \mathrm{~d}$ MC1-positive cells). All values are expressed as mean \pm SEM. expression of tau during the progression of AD pathology. Nevertheless, in these models, differences in the function and aggregation propensity between wild-type and mutant tau may not accurately predict the molecular and cellular mechanisms that drive tau pathology in $\mathrm{AD}$ (Hong et al., 1998; Combs and Gamblin, 2012). Therefore, we have focused our research on the development of a more relevant model of sporadic tauopathies to study the specific events involved in the progression of the pathology. The data presented here demonstrate that wild-type fulllength human tau oligomers induce tau aggregation in human neurons with no tau mutations or overexpression. Additionally, changes in phosphorylated tau and tau conformation, consistent with $\mathrm{AD}$ tau pathology, were also observed. Importantly, chronic evaluation of tau oligomer-seeded neurons revealed a progressive deficit in neurite outgrowth, loss of synapses, deficits in endogenous calcium homeostasis, abnormal neurotransmitter release, and ultimately neuronal cell death. Notably, neurodegeneration was exacerbated in the neuronal population exhibiting pathological tau aggregates (MC1-positive neurons). These findings suggest that the pathological changes induced by internalized tau oligomers can lead to neurodegeneration and neuronal death in human neurons. Together these data are consistent with previous human studies demonstrating a correlation between the spreading of tau pathology and neurodegeneration in $\mathrm{AD}$ (Delacourte et al., 2002a, 2002b).

The majority of studies to date have demonstrated the seeding property of recombinant tau fibrils, which are made of large forms of insoluble tau aggregates (Guo and Lee, 2011; Santa- 
Maria et al., 2012; Holmes et al., 2013; Iba et al., 2013). However, increasing evidence suggest that prefibrillar oligomers, rather than mature tau fibrils, are the pathogenic species in AD (Kayed et al., 2003; Lasagna-Reeves et al., 2011, 2012a, 2012b; Patterson et al., 2011). The levels of tau oligomers were found to be significantly increased in AD brains early in disease, before the appearance of NFTs or clinical symptoms (Gerson and Kayed, 2013). In wild-type mice, injection of tau oligomers, but not tau monomers or fibrils, induced memory impairments and synaptic dysfunction (Lasagna-Reeves et al., 2011, 2012a, 2012b; Patterson et al., 2011).

In previous experiments, only low molecular weight aggregates and short fibrils, rather than long fibrils, were taken up by primary neurons, suggesting that tau internalization is dependent on the conformation and size of the aggregates (Wu et al., 2013). Our data are consistent with these initial findings that tau fibrils are not readily taken up by hiPSC neurons. It is also noteworthy that in certain tau models the fibrils were sonicated to produce fragmented species before brain injection or cell treatment (Guo and Lee, 2013; Iba et al., 2013). Therefore, it is likely that the sonicated tau fibrils also contained tau in oligomeric forms, which may explain why seeding and propagation of tau aggregation was successful. Indeed, in a recent study, it was demonstrated that sonication of recombinant fibrils results in a mixed population of various sizes of tau assemblies, from which tau trimers were the minimal-size species able to induce intracellular aggregation. These data further confirm the pathogenic seeding properties of small tau oligomeric species also found in our oligomer preparations (Mirbaha et al., 2015). In the present study, the pathogenic properties of tau oligomers are also supported by the observation that even small amounts of tau oligomeric species can be internalized and transported by the human neurons to propagate and seed the aggregation of endogenous tau. Tau oligomer trafficking through neuronal axons was demonstrated using microfluidic chambers. The recruitment of endogenous tau was determined by using an AlphaLISA aggregation assay. The observed intracellular tau aggregates formed after oligomer treatment share key features with mature NFTs from AD brains. Aggregates were intensely stained with ThS and immunostained with specific antibodies detecting pathological tau conformation (MC1) and tau phosphorylation. Tau oligomers also induced accumulation of insoluble hyperphosphorylated tau species observed with immunoblotting. Together, these results showed that tau oligomers are capable of propagating tau aggregation in human neurons in a manner consistent with the hypothesis of tau spreading in $\mathrm{AD}$.

Several studies have demonstrated that exogenously applied tau protein is internalized by cells. However, the precise cellular and molecular mechanisms that underlie this process are not well understood (Frost et al., 2009; Guo and Lee, 2011, 2013; Wu et al., 2013). Recently it was suggested that the internalization process may occur through a mechanism of bulk endocytosis or macropinocytosis involving HSPGs (Holmes and Diamond, 2012; Wu et al., 2013). In the present study, tau oligomer seeds were prepared from recombinant tau labeled with a Cy3 dye. Cy3-tau conjugates, in combination with a background suppressor that depletes extracellular fluorescence, enabled us to differentiate externally added tau oligomers, which bind to the cell surface, from those internalized in the neurons. The increase in fluorescence at $1 \mathrm{~h}$ after Cy3-tau oligomer treatment suggests that tau oligomers are rapidly internalized by neurons. Moreover, pretreatment of the neurons with heparinase can effectively reduce the amount of internalized tau oligomers, suggesting the role of HSPGs in the process of internalization in human neurons. Recent studies have also reported that internalized tau aggregates colocalize with endosomal and lysosomal markers (Wu et al., 2013). This colocalization of internalized tau recombinant protein with lysosomal markers suggests that the autophagy pathway might be responsible for their degradation. Consistent with this, our data showed that tau seed-induced intracellular aggregates could be reduced by enhancing autophagy with rapamycin.

In our study, tau oligomer treatment induced a progressive neuronal loss and deficit of neurite outgrowth that could replicate the neurodegenerative process observed in animal models of tauopathy (Lee et al., 2005; Yoshiyama et al., 2007; LasagnaReeves et al., 2011; de Calignon et al., 2012; Cohen et al., 2013), as well as in AD patients (Braak and Braak, 1995). In addition, the loss of synapses that followed the accumulation of tau aggregates could reflect a general impairment of neuronal function that precedes cell death. Indeed, neurons that were treated with tau oligomers showed abnormal changes in GABA release, whereas glutamate release was not changed. This finding could suggest that GABAergic hiPSC neurons are preferentially vulnerable to tau oligomer seeds. Similarly, it was interesting that only a certain population of cells ( $\sim 15-50 \%$ of cells depending on the concentration of tau seeds) were positive for pathological MC1-tau. Meanwhile most cells were capable of internalizing the tau oligomers based on $\mathrm{Cy} 3$ conjugate fluorescence. Further research is required to investigate and characterize the specific populations of affected human neurons and may provide a better understanding of cellular pathways implicated in pathogenic tau propagation and transmission.

Last, tau oligomer treatment induced changes in intracellular $\mathrm{Ca}^{2+}$ levels that coincided with neurite retraction, loss of synapses, and imbalanced neurotransmitter release. These findings suggest that pathological tau oligomers may affect regulation of calcium homeostasis. This is consistent with previous studies that have reported an association between tau pathology formation and aberrant increases in $\mathrm{Ca}^{2+}$ levels in the brains of $\mathrm{AD}$ patients and in in vitro models (McKee et al., 1990; Nixon, 2003; Bezprozvanny and Mattson, 2008). Together, these data reveal several pathological changes to intracellular functions that are capable of compromising neuronal function and highlight the need for further research to elucidate novel approaches for intervention.

It was notable that the administration of tau monomers did not induce changes in tau pathology or cell morphology, despite being internalized by neurons comparably to tau oligomers. Addition of full-length human tau to human neurons may not have disturbed cellular homeostasis, and suggests the cells are capable of effectively coping with the excess monomeric tau through degradation. In support of this, it was recently demonstrated that internalized recombinant tau monomers failed to induce aggregation in cell lines overexpressing P301S mutant tau (Falcon et al., 2015).

In conclusion, the findings from the present study demonstrate that tau oligomers, and not monomeric tau, can act as aggregation seeds that can ultimately compromise neuronal integrity and induce the accumulation of pathogenic tau. This tauseeding model in hiPSC neurons opens up novel avenues of research and may be useful in uncovering the mechanisms responsible for the transmission of tau pathology and its deleterious effects on neuronal viability and function.

\section{References}

Alhebshi AH, Odawara A, Gotoh M, Suzuki I (2014) Thymoquinone protects cultured hippocampal and human induced pluripotent stem cells- 
derived neurons against $\alpha$-synuclein-induced synapse damage. Neurosci Lett 570:126-131. CrossRef Medline

Arriagada PV, Growdon JH, Hedley-Whyte ET, Hyman BT (1992) Neurofibrillary tangles but not senile plaques parallel duration and severity of Alzheimer's disease. Neurology 42:631-639. CrossRef Medline

Ballatore C, Lee VM, Trojanowski JQ (2007) Tau-mediated neurodegeneration in Alzheimer's disease and related disorders. Nat Rev Neurosci 8:663-672. CrossRef Medline

Berger Z, Ravikumar B, Menzies FM, Oroz LG, Underwood BR, Pangalos MN, Schmitt I, Wullner U, Evert BO, O’Kane CJ, Rubinsztein DC (2006) Rapamycin alleviates toxicity of different aggregate-prone proteins. Hum Mol Genet 15:433-442. Medline

Berger Z, Roder H, Hanna A, Carlson A, Rangachari V, Yue M, Wszolek Z, Ashe K, Knight J, Dickson D, Andorfer C, Rosenberry TL, Lewis J, Hutton M, Janus C (2007) Accumulation of pathological tau species and memory loss in a conditional model of tauopathy. J Neurosci 27:3650-3662. CrossRef Medline

Bezprozvanny I, Mattson MP (2008) Neuronal calcium mishandling and the pathogenesis of Alzheimer's disease. Trends Neurosci 31:454-463. CrossRef Medline

Bich C, Maedler S, Chiesa K, DeGiacomo F, Bogliotti N, Zenobi R (2010) Reactivity and applications of new amine reactive cross-linkers for mass spectrometric detection of protein-protein complexes. Anal Chem 82: 172-179. CrossRef Medline

Braak H, Braak E (1995) Staging of Alzheimer's disease-related neurofibrillary changes. Neurobiol Aging 16:271-278; discussion 278-284. Medline

Chai X, Dage JL, Citron M (2012) Constitutive secretion of tau protein by an unconventional mechanism. Neurobiol Dis 48:356-366. CrossRef Medline

Clavaguera F, Bolmont T, Crowther RA, Abramowski D, Frank S, Probst A, Fraser G, Stalder AK, Beibel M, Staufenbiel M, Jucker M, Goedert M, Tolnay M (2009) Transmission and spreading of tauopathy in transgenic mouse brain. Nat Cell Biol 11:909-913. CrossRef Medline

Clavaguera F, Akatsu H, Fraser G, Crowther RA, Frank S, Hench J, Probst A, Winkler DT, Reichwald J, Staufenbiel M, Ghetti B, Goedert M, Tolnay M (2013) Brain homogenates from human tauopathies induce tau inclusions in mouse brain. Proc Natl Acad Sci U S A 110:9535-9540. CrossRef Medline

Clavaguera F, Grueninger F, Tolnay M (2014) Intercellular transfer of tau aggregates and spreading of tau pathology: implications for therapeutic strategies. Neuropharmacology 76:9-15. CrossRef Medline

Cohen RM, Rezai-Zadeh K, Weitz TM, Rentsendorj A, Gate D, Spivak I, Bholat Y, Vasilevko V, Glabe CG, Breunig JJ, Rakic P, Davtyan H, Agadjanyan MG, Kepe V, Barrio JR, Bannykh S, Szekely CA, Pechnick RN, Town T (2013) A transgenic Alzheimer rat with plaques, tau pathology, behavioral impairment, oligomeric a $\beta$, and frank neuronal loss. J Neurosci 33:6245-6256. CrossRef Medline

Combs B, Gamblin TC (2012) FTDP-17 tau mutations induce distinct effects on aggregation and microtubule interactions. Biochemistry 51: 8597-8607. CrossRef Medline

Dage JL, Colvin EM, Fouillet A, Langron E, Roell WC, Li J, Mathur SX, Mogg AJ, Schmitt MG, Felder CC, Merchant KM, Isaac J, Broad LM, Sher E, Ursu D (2014) Pharmacological characterisation of ligand- and voltagegated ion channels expressed in human iPSC-derived forebrain neurons. Psychopharmacology 231:1105-1124. CrossRef Medline

de Calignon A, Polydoro M, Suárez-Calvet M, William C, Adamowicz DH, Kopeikina KJ, Pitstick R, Sahara N, Ashe KH, Carlson GA, Spires-Jones TL, Hyman BT (2012) Propagation of tau pathology in a model of early Alzheimer's disease. Neuron 73:685-697. CrossRef Medline

Delacourte A, Sergeant N, Champain D, Wattez A, Maurage CA, Lebert F, Pasquier F, David JP (2002a) Nonoverlapping but synergetic tau and APP pathologies in sporadic Alzheimer's disease. Neurology 59:398-407. CrossRef Medline

Delacourte A, Sergeant N, Wattez A, Maurage CA, Lebert F, Pasquier F, David JP (2002b) Tau aggregation in the hippocampal formation: an ageing or a pathological process? Exp Gerontol 37:1291-1296. CrossRef Medline

Falcon B, Cavallini A, Angers R, Glover S, Murray TK, Barnham L, Jackson S, O’Neill MJ, Isaacs AM, Hutton ML, Szekeres PG, Goedert M, Bose S (2015) Conformation determines the seeding potencies of native and recombinant tau aggregates. J Biol Chem 290:1049-1065. CrossRef Medline

Fletcher TL, Cameron P, De Camilli P, Banker G (1991) The distribution of synapsin I and synaptophysin in hippocampal neurons developing in culture. J Neurosci 11:1617-1626. Medline

Frederick C, Ando K, Leroy K, Héraud C, Suain V, Buée L, Brion JP (2015) Rapamycin ester analog CCI-779/temsirolimus alleviates tau pathology and improves motor deficit in mutant tau transgenic mice. J Alzheimers Dis 44:1145-1156. CrossRef Medline

Friedman LG, Qureshi YH, Yu WH (2015) Promoting autophagic clearance: viable therapeutic targets in Alzheimer's disease. Neurotherapeutics 12:94-108. CrossRef Medline

Frost B, Jacks RL, Diamond MI (2009) Propagation of tau misfolding from the outside to the inside of a cell. J Biol Chem 284:12845-12852. CrossRef Medline

Gerson JE, Kayed R (2013) Formation and propagation of tau oligomeric seeds. Front Neurol 4:93. CrossRef Medline

Guo JL, Lee VM (2011) Seeding of normal tau by pathological tau conformers drives pathogenesis of Alzheimer-like tangles. J Biol Chem 286:1531715331. CrossRef Medline

Guo JL, Lee VM (2013) Neurofibrillary tangle-like tau pathology induced by synthetic tau fibrils in primary neurons over-expressing mutant tau. FEBS Lett 587:717-723. CrossRef Medline

Haythornthwaite A, Stoelzle S, Hasler A, Kiss A, Mosbacher J, George M, Brüggemann A, Fertig N (2012) Characterizing human ion channels in induced pluripotent stem cell-derived neurons. J Biomol Screen 17:12641272. CrossRef Medline

Holmes BB, Diamond MI (2012) Cellular mechanisms of protein aggregate propagation. Curr Opin Neurol 25:721-726. CrossRef Medline

Holmes BB, DeVos SL, Kfoury N, Li M, Jacks R, Yanamandra K, Ouidja MO, Brodsky FM, Marasa J, Bagchi DP, Kotzbauer PT, Miller TM, PapyGarcia D, Diamond MI (2013) Heparan sulfate proteoglycans mediate internalization and propagation of specific proteopathic seeds. Proc Natl Acad Sci U S A 110:E3138-E3147. CrossRef Medline

Hong M, Zhukareva V, Vogelsberg-Ragaglia V, Wszolek Z, Reed L, Miller BI, Geschwind DH, Bird TD, McKeel D, Goate A, Morris JC, Wilhelmsen KC, Schellenberg GD, Trojanowski JQ, Lee VM (1998) Mutation-specific functional impairments in distinct tau isoforms of hereditary FTDP-17. Science 282:1914-1917. CrossRef Medline

Iba M, Guo JL, McBride JD, Zhang B, Trojanowski JQ, Lee VM (2013) Synthetic tau fibrils mediate transmission of neurofibrillary tangles in a transgenic mouse model of Alzheimer's-like tauopathy. J Neurosci 33: 1024-1037. CrossRef Medline

Jameson DM, Croney JC (2003) Fluorescence polarization: past, present and future. Comb Chem High Throughput Screen 6:167-173. CrossRef Medline

Jameson DM, Seifried SE (1999) Quantification of protein-protein interactions using fluorescence polarization. Methods 19:222-233. CrossRef Medline

Jicha GA, Bowser R, Kazam IG, Davies P (1997) Alz-50 and MC-1, a new monoclonal antibody raised to paired helical filaments, recognize conformational epitopes on recombinant tau. J Neurosci Res 48:128-132. CrossRef Medline

Jucker M, Walker LC (2013) Self-propagation of pathogenic protein aggregates in neurodegenerative diseases. Nature 501:45-51. CrossRef Medline

Kabeya Y, Mizushima N, Ueno T, Yamamoto A, Kirisako T, Noda T, Kominami E, Ohsumi Y, Yoshimori T (2000) LC3, a mammalian homologue of yeast Apg8p, is localized in autophagosome membranes after processing. EMBO J 19:5720-5728. CrossRef Medline

Kayed R, Head E, Thompson JL, McIntire TM, Milton SC, Cotman CW, Glabe CG (2003) Common structure of soluble amyloid oligomers implies common mechanism of pathogenesis. Science 300:486-489. CrossRef Medline

Kfoury N, Holmes BB, Jiang H, Holtzman DM, Diamond MI (2012) Transcellular propagation of tau aggregation by fibrillar species. J Biol Chem 287:19440-19451. CrossRef Medline

Klionsky DJ, Abdalla FC, Abeliovich H, Abraham RT, Acevedo-Arozena A, Adeli K, Agholme L, Agnello M, Agostinis P, Aguirre-Ghiso JA, Ahn HJ, Ait-Mohamed O, Ait-Si-Ali S, Akematsu T, Akira S, Al-Younes HM, AlZeer MA, Albert ML, Albin RL, Alegre-Abarrategui J et al. (2012) Guidelines for the use and interpretation of assays for monitoring autophagy. Autophagy 8:445-544. CrossRef Medline

Lasagna-Reeves CA, Castillo-Carranza DL, Sengupta U, Clos AL, Jackson GR, Kayed R (2011) Tau oligomers impair memory and induce synaptic and 
mitochondrial dysfunction in wild-type mice. Mol Neurodegener 6:39. CrossRef Medline

Lasagna-Reeves CA, Castillo-Carranza DL, Sengupta U, Sarmiento J, Troncoso J, Jackson GR, Kayed R (2012a) Identification of oligomers at early stages of tau aggregation in Alzheimer's disease. FASEB J 26:1946-1959. CrossRef Medline

Lasagna-Reeves CA, Castillo-Carranza DL, Sengupta U, Guerrero-Munoz MJ, Kiritoshi T, Neugebauer V, Jackson GR, Kayed R (2012b) Alzheimer brain-derived tau oligomers propagate pathology from endogenous tau. Sci Rep 2:700. CrossRef Medline

Lee VM, Kenyon TK, Trojanowski JQ (2005) Transgenic animal models of tauopathies. Biochim Biophys Acta 1739:251-259. CrossRef Medline

LeVine H 3rd (1999) Quantification of beta-sheet amyloid fibril structures with thioflavin T. Methods Enzymol 309:274-284. CrossRef Medline

Li W, Lee VM (2006) Characterization of two VQIXXK motifs for tau fibrillization in vitro. Biochemistry 45:15692-15701. CrossRef Medline

Maeda S, Sahara N, Saito Y, Murayama S, Ikai A, Takashima A (2006) Increased levels of granular tau oligomers: an early sign of brain aging and Alzheimer's disease. Neurosci Res 54:197-201. CrossRef Medline

Mandelkow EM, Mandelkow E (2012) Biochemistry and cell biology of tau protein in neurofibrillary degeneration. Cold Spring Harb Perspect Med 2:a006247. CrossRef Medline

McKee AC, Kosik KS, Kennedy MB, Kowall NW (1990) Hippocampal neurons predisposed to neurofibrillary tangle formation are enriched in type II calcium/calmodulin-dependent protein kinase. J Neuropathol Exp Neurol 49:49-63. CrossRef Medline

Mirbaha H, Holmes BB, Sanders DW, Bieschke J, Diamond MI (2015) Tau trimers are the minimal propagation unit spontaneously internalized to seed intracellular aggregation. J Biol Chem 290:14893-14903. CrossRef Medline

Mizushima N (2011) Autophagy in protein and organelle turnover. Cold Spring Harb Symp Quant Biol 76:397-402. CrossRef Medline

Moerke NJ (2009) Fluorescence polarization (FP) assays for monitoring peptide-protein or nucleic acid-protein binding. Curr Protoc Chem Biol 1:1-15. CrossRef Medline

Nazabal A, Wenzel RJ, Zenobi R (2006) Immunoassays with direct mass spectrometric detection. Anal Chem 78:3562-3570. CrossRef Medline

Nixon RA (2003) The calpains in aging and aging-related diseases. Ageing Res Rev 2:407-418. CrossRef Medline

Patterson KR, Remmers C, Fu Y, Brooker S, Kanaan NM, Vana L, Ward S, Reyes JF, Philibert K, Glucksman MJ, Binder LI (2011) Characterization of prefibrillar tau oligomers in vitro and in Alzheimer disease. J Biol Chem 286:23063-23076. CrossRef Medline

Pooler AM, Phillips EC, Lau DH, Noble W, Hanger DP (2013) Physiological release of endogenous tau is stimulated by neuronal activity. EMBO Rep 14:389-394. CrossRef Medline

Saman S, Kim W, Raya M, Visnick Y, Miro S, Saman S, Jackson B, McKee AC, Alvarez VE, Lee NC, Hall GF (2012) Exosome-associated tau is secreted in tauopathy models and is selectively phosphorylated in cerebrospinal fluid in early Alzheimer disease. J Biol Chem 287:3842-3849. CrossRef Medline

Santa-María I, Pérez M, Hernández F, Avila J, Moreno FJ (2006) Characteristics of the binding of thioflavin $\mathrm{S}$ to tau paired helical filaments. J Alzheimers Dis 9:279-285. Medline

Santa-Maria I, Varghese M, Ksiezak-Reding H, Dzhun A, Wang J, Pasinetti GM (2012) Paired helical filaments from Alzheimer disease brain induce intracellular accumulation of tau protein in aggresomes. J Biol Chem 287:20522-20533. CrossRef Medline

Simón D, García-García E, Royo F, Falcón-Pérez JM, Avila J (2012) Proteostasis of tau. Tau overexpression results in its secretion via membrane vesicles. FEBS Lett 586:47-54. CrossRef Medline

Supnet C, Bezprozvanny I (2010) The dysregulation of intracellular calcium in Alzheimer disease. Cell Calcium 47:183-189. CrossRef Medline

Taylor AM, Blurton-Jones M, Rhee SW, Cribbs DH, Cotman CW, Jeon NL (2005) A microfluidic culture platform for CNS axonal injury, regeneration and transport. Nat Methods 2:599-605. CrossRef Medline

Weaver CL, Espinoza M, Kress Y, Davies P (2000) Conformational change as one of the earliest alterations of tau in Alzheimer's disease. Neurobiol Aging 21:719-727. CrossRef Medline

Wu JW, Herman M, Liu L, Simoes S, Acker CM, Figueroa H, Steinberg JI, Margittai M, Kayed R, Zurzolo C, Di Paolo G, Duff KE (2013) Small misfolded tau species are internalized via bulk endocytosis and anterogradely and retrogradely transported in neurons. J Biol Chem 288:18561870. CrossRef Medline

Xu X, Lei Y, Luo J, Wang J, Zhang S, Yang XJ, Sun M, Nuwaysir E, Fan G, Zhao J, Lei L, Zhong Z (2013) Prevention of $\beta$-amyloid induced toxicity in human iPS cell-derived neurons by inhibition of cyclin-dependent kinases and associated cell cycle events. Stem Cell Res 10:213-227. CrossRef Medline

Yoshiyama Y, Higuchi M, Zhang B, Huang SM, Iwata N, Saido TC, Maeda J, Suhara T, Trojanowski JQ, Lee VM (2007) Synapse loss and microglial activation precede tangles in a P301S tauopathy mouse model. Neuron 53:337-351. CrossRef Medline 